\title{
THE BOUNDED VECTOR MEASURE ASSOCIATED TO A CONICAL MEASURE AND PETTIS DIFFERENTIABILITY
}

\author{
L. RODRIGUEZ-PIAZZA and M. C. ROMERO-MORENO
}

(Received 11 August 1999; revised 19 June 2000)

Communicated by P. G. Dodds

\begin{abstract}
Let $X$ be a locally convex space. Kluvánek associated to each $X$-valued countably additive vector measure a conical measure on $X$; this can also be done for finitely additive bounded vector measures. We prove that every conical measure $u$ on $X$, whose associated zonoform $K_{u}$ is contained in $X$, is associated to a bounded additive vector measure $\sigma(u)$ defined on $X$, and satisfying $\sigma(u)(H) \in H$, for every finite intersection $H$ of closed half-spaces. When $X$ is a complete weak space, we prove that $\sigma(u)$ is countably additive. This allows us to recover two results of Kluvánek: for any $X$, every conical measure $u$ on it with $K_{u} \subseteq X$ is associated to a countably additive $X$-valued vector measure; and every conical measure on a complete weak space is localizable. When $X$ is a Banach space, we prove that $\sigma(u)$ is countably additive if and only if $u$ is the conical measure associated to a Pettis differentiable vector measure.
\end{abstract}

2000 Mathematics subject classification: primary 46G10, 28B05, 47D50.

Keywords and phrases: vector measures, conical measures, ranges of vector measures, Pettis integral.

\section{Introduction}

In [K1] Kluvánek introduced the concept of the conical measure associated to a countably additive vector measure to characterize the closed convex hull of its range. Many properties of a vector measure can be determined from the corresponding associated conical measure. For instance, in the framework of Banach spaces, having finite variation, having $\sigma$-finite variation, having a Bochner derivative, or having $p$ summing integration operator are properties of a vector measure characterized in terms of its associated conical measure (see [RR], where these characterizations are used to prove that these properties are determined by the range).

Research supported in part by DGES grant \#PB96-1327.

(C) 2001 Australian Mathematical Society 0263-6115/2001 \$A2.00+0.00 
Conversely, vector measures are used as a tool to study conical measures on a locally convex space $X$; this is due to the fact, proved by Kluvánek [K1], that every conical measure $u$ on $X$, whose associated zonoform $K_{u}$ (see definition in Section 1) is contained in $X$, is the associated conical measure to an $X$-valued vector measure $F$. We prove this fact in Corollary 2.4; Kluvánek's proof is purely existential and does not show any relationship between $u$ and the vector measure $F$ or the measurable space where $F$ is defined. Finding a more explicit construction of the vector measure $F$ was our first motivation for this work.

The most natural requirement for $F$ is to define it on a $\sigma$-algebra of subsets of $X$ containing the closed convex cones, and with the condition $F(C) \in C$ for every closed convex cone $C$. This can be done in finite dimension, but it is not always possible in general. Even if we consider the smaller $\sigma$-algebra generated by the closed half-spaces, we prove that there would exist only one way to construct such an $F$ (uniqueness in Theorem 1.2), and this way does not give countably additiveness in general (Example 3.1). This is the reason why we consider finitely additive measures.

Using the same scheme as Kluvánek, we associate to every $X$-valued finitely additive bounded measure $F$ a conical measure $\Delta(F)$ on $X$ with the property that the closed convex hull of the range of $F$ is the zonoform associated to $\Delta(F)$. This is done in Section 1. The main effort in this section is devoted to obtaining a natural converse of this result. In Theorem 1.2 we prove that, given a conical measure $u$ on $X$ whose associated zonoform $K_{u}$ is contained in $X$, there exists a bounded finitely additive measure $\sigma(u)$, defined on the algebra of subsets of $X$ generated by the closed half-spaces, such that $\sigma(u)(H) \in H \cup\{0\}$, for every finite intersection $H$ of closed or open half-spaces.

In Section 2 we deal with the case where $X$ is a weak complete space. Then for every conical measure $u$ on $X, K_{u} \subset X, \sigma(u)$ is defined, and moreover, $\sigma(u)$ is countably additive (Theorem 2.3). This allows us to extend $\sigma(u)$ to a countably additive measure on the $\sigma$-algebra generated by the closed half-spaces. Corollary 2.4 is obtained considering, for any locally convex space, its weak completion. We finish this section giving a new proof of another result of Kluvánek: every conical measure on a weak complete space is localizable (Theorem 2.8).

In the last section we consider Banach spaces. We prove (Theorem 3.3) that, for a Banach space $X, \sigma(u)$ is countably additive if and only if there exists a Pettis differentiable $X$-valued measure $F$ such that $u$ is the conical measure associated to $F$. An analogous condition (using the bidual $X^{* *}$ of $X$ ) is obtained in Theorem 3.4 to characterize when $u$ is the conical measure associated to a vector measure with $\sigma$-finite variation.

Throughout the paper our locally convex space and measure theory terminology follows [BB, C, DU]. We only consider real locally convex spaces. Let $(\Omega, \mathscr{F})$ be a pair, where $\mathscr{F}$ is a field of subsets of a set $\Omega$. We denote by ba $(\Omega, \mathscr{F})$ the space of all 
bounded additive real-valued measures on $\mathscr{F}$ and by ca $(\Omega, \Sigma)$ the space of countably additive real-valued measures on a $\sigma$-field $\Sigma$. If $\mu$ is a measure in one of these spaces, $\mu \geq 0$ means that $\mu(A) \geq 0$ for every $A \in \mathscr{F}$ (for every $A \in \Sigma$ ). With this ordering $\mathrm{ba}(\Omega, \mathscr{F})$ and $\mathrm{ca}(\Omega, \Sigma)$ become vector lattices. In particular, the positive part of $\mu$, $\mu^{+}$, is given by

$$
\mu^{+}(A)=\sup \{\mu(B): B \subseteq A, B \in \mathscr{F}\} \quad \text { for every } A \in \mathscr{F}
$$

and the negative part $\mu^{-}$by

$$
\mu^{-}(A)=-\inf \{\mu(B): B \subseteq A, B \in \mathscr{F}\} \quad \text { for every } A \in \mathscr{F} .
$$

The variation of $\mu$, denoted by $|\mu|$, is given by $|\mu|=\mu^{+}+\mu^{-}$.

Let $X$ be a Hausdorff locally convex space, with the topological dual space $X^{*}$. For us a vector measure is an additive function $F: \mathscr{F} \rightarrow X$ defined on the space $(\Omega, \mathscr{F})$ with values in $X$. If $E \in \mathscr{F}$, the vector measure $F_{E}: \mathscr{F} \rightarrow X$ is defined by $F_{E}(A)=F(E \cap A)$ for every $A \in \mathscr{F}$.

The range of $F$ is denoted by $\operatorname{rg} F$, that is, $\operatorname{rg} F=\{F(A): A \in \mathscr{F}\}$. We say that a vector measure $F$ is bounded if $x^{*} F \in \operatorname{ba}(\Omega, \mathscr{F})$ for every $x^{*} \in X^{*}$, that is, if $\operatorname{rg} F$ is a bounded set in $X$, thanks to the Mackey theorem.

By a countably additive vector measure $F$ on an algebra $\mathscr{F}$ we understand a vector measure such that $F\left(\bigcup_{n=1}^{\infty} A_{n}\right)=\sum_{n=1}^{\infty} F\left(A_{n}\right)$ for all sequences $\left(A_{n}\right)$ of pairwise disjoint members of $\mathscr{F}$ such that $\bigcup_{n=1}^{\infty} A_{n} \in \mathscr{F}$. This is equivalent to the following condition: $F$ is additive and if $\left(A_{n}\right)$ is a sequence of sets in $\mathscr{F}$ decreasing to the empty set, then $F\left(A_{n}\right)$ converges to 0 in $X$. If in addition there exists a weakly compact convex set $W$ such that $\operatorname{rg} F \subseteq W$, then $F$ has a (unique) countably additive extension to the $\sigma$-algebra $\Sigma$ generated by $\mathscr{F}$. To show this fact, apply the Hahn extension theorem to each scalar measure $x^{*} F$ and extended it to a countably additive measure $\mu_{x^{*}}$. Thanks to the weak compactness, it is easy to see that for every $A \in \Sigma$ there is $x_{A} \in W$ such that $x^{*}\left(x_{A}\right)=\mu_{x^{*}}(A)$, for every $x^{*} \in X^{*}$. The mapping $A \mapsto x_{A}$ is weakly countably additive and, by the Orlicz-Pettis theorem [KK, page 4], it is countably additive (see also [DU, page 27] for the Banach space case).

If $X$ is a Banach space, $F$ is said to be strongly additive whenever given a sequence $\left(E_{n}\right)$ of pairwise disjoint members of $\mathscr{F}$, the series $\sum_{n=1}^{\infty} F\left(E_{n}\right)$ converges in norm. In this case, $F$ is strongly additive if and only if $\overline{c o}(\mathrm{rg} F)$ is a weakly compact set in $X$ (see [DU, page 27]).

\section{Conical measures and bounded vector measures}

In [K1], Kluvánek introduced the conical measure associated to a countably additive vector measure to study the closed convex hull of its range. We work in a wider context 
of vector measures which are bounded. Let us recall some basic facts and definitions about conical measures.

Given a Hausdorff locally convex space $X, h(X)$ stands for the smallest vector lattice of functions on $X$ with respect to the pointwise order and the linear operations that contains $X^{*}$. Every element of $h(X)$ can be written in the form

$$
z^{*}=\bigvee_{i=1}^{n} x_{i}^{*}-\bigvee_{i=n+1}^{m} x_{i}^{*}
$$

where $x_{i}^{*} \in X^{*}$ for $i=1, \ldots, m$, and $\vee(\wedge)$ denote the least upper (greatest lower) bound in a lattice; which in this case is a pointwise maximum (minimum) of functions.

A conical measure on $X$ is a positive linear functional on $h(X)$. The set of all conical measures over $X$ is denoted $M^{+}(X)$. It is a complete lattice with respect to the order $v \leq u$ if $v\left(z^{*}\right) \leq u\left(z^{*}\right)$, for every $z^{*} \in h(X), z^{*} \geq 0$. We refer to [C, Sections 38-40] for these and more facts about conical measures. In particular, we have the Riesz decomposition [C, 10.5]: given $u_{1}, u_{2}$ and $v$ in $M^{+}(X)$ such that $v \leq u_{1}+u_{2}$, there exist $v_{1}$ and $v_{2}$ in $M^{+}(X)$ with $v=v_{1}+v_{2}, v_{1} \leq u_{1}$ and $v_{2} \leq u_{2}$. On $M^{+}(X)$ we consider the topology of pointwise convergence, that is, a net $\left(u_{\delta}\right)_{\delta}$ in $M^{+}(X)$ converges to $u \in M^{+}(X)$ if $u_{\delta}\left(z^{*}\right)$ converges to $u\left(z^{*}\right)$ for every $z^{*} \in h(X)$. For this topology, given $u \in M^{+}(X)$, the set $L=\left\{v \in M^{+}(X): 0 \leq v \leq u\right\}$ is compact.

In the sequel we also consider conical measures on the weak completion of $X$. This weak completion, that we denote by $\widehat{X}$, turns out to be the algebraic dual of $X^{*}$, that is, $\widehat{X}=\left\{f: X^{*} \rightarrow \mathbb{R}, f\right.$ linear $\}$. When considering the weak topology $\sigma\left(\widehat{X}, X^{*}\right), X$ is a dense subset of $\widehat{X}$ and $(\widehat{X})^{*}=X^{*}$, so we can identify $h(X)$ and $h(\widehat{X})$ and conical measures are unaffected.

The resultant of a conical measure $u$ is defined as the vector $r(u)$ in $\widehat{X}$ satisfying $u\left(x^{*}\right)=x^{*}(r(u))$ for all $x^{*} \in X^{*}$. The zonoform associated to $u$ is the set in $\widehat{X}$, $K_{u}=\left\{r(v): v \in M^{+}(X), 0 \leq v \leq u\right\}$. By considering $\widehat{X}$, the existence of $r(v)$ is always ensured.

Let us suppose now that $F:(\Omega, \mathscr{F}) \rightarrow X$ is a bounded vector measure. It defines a linear map from $X^{*}$ to ba $(\Omega, \mathscr{F})$ sending each $x^{*}$ to $x^{*} F$. This map can be extended to a lattice homomorphism $\Phi_{F}$ from $h(X)$ to ba $(\Omega, \mathscr{F})$, such that for every $z^{*} \in h(X)$ of the form (1),

$$
\Phi_{F}\left(z^{*}\right)=\bigvee_{i=1}^{n} x_{i}^{*} F-\bigvee_{i=n+1}^{m} x_{i}^{*} F .
$$

The conical measure $\Delta(F)$ associated to $F$ is defined by

$$
\Delta(F)\left(z^{*}\right)=\Phi_{F}\left(z^{*}\right)(\Omega), \quad \text { for every } z^{*} \in h(X) .
$$


In this case, $r(\Delta(F))=F(\Omega)$. The original definition of Kluvánek in [K1] used the lattice structure of $\mathrm{ca}(\Omega, \Sigma)$ for countably additive vector measures, instead of that of $\mathrm{ba}(\Omega, \mathscr{F})$.

Let us observe first that if $F$ is a bounded vector measure with values in a locally convex space $X$, if $v \in M^{+}(X), 0 \leq v \leq \Delta(F)$, then the resultant of $v$ always exists as an element of $X^{* *}$, the topological bidual of $X$. To show this, observe that for every $x^{*} \in X^{*}$,

$$
\begin{aligned}
\left|v\left(x^{*}\right)\right| & \leq v\left(\left(x^{*}\right)^{+}\right)+v\left(\left(x^{*}\right)^{-}\right) \leq \Delta(F)\left(\left(x^{*}\right)^{+}\right)+\Delta(F)\left(\left(x^{*}\right)^{-}\right) \\
& =\left(x^{*} F\right)^{+}(\Omega)+\left(x^{*} F\right)^{-}(\Omega) \leq 2 \sup \left\{\left|x^{*}(x)\right|: x \in \operatorname{rg} F\right\} .
\end{aligned}
$$

This implies, since $\operatorname{rg} F$ is a bounded set, that $r(v)$ is continuous for the topology $\beta\left(X^{*}, X\right)$ in $X^{*}$, that is, $r(v) \in X^{* *}$. This means that $K_{\Delta(F)}$ is contained in $X^{* *}$ in this case. It is not a general fact for a conical measure $u$ that $r(u) \in X^{* *}$ (see [C, vol. 3, page 4]).

Kluvánek identified in [K1] the closed convex hull of the range of $F$ with the zonoform associated to $\Delta(F)$ when $F$ is countably additive. We show the corresponding fact in the following result. We denote by $\tau$ the weak topology $\sigma\left(\widehat{X}, X^{*}\right)$ on $\widehat{X}$.

THEOREM 1.1. If $F: \mathscr{F} \rightarrow X$ is a bounded vector measure with values in a locally convex space $X$ and $\Delta(F)$ is its associated conical measure, then $\overline{\mathrm{co}}^{\tau}(\mathrm{rg} F)=K_{\Delta(F)}$. In addition, $K_{\Delta(F)} \subseteq X$ if and only if $\overline{\mathrm{co}}(\mathrm{rg} F)$ is weakly compact in $X$ if and only if there exists a weakly compact convex set $W$ in $X$ such that $\operatorname{rg} F \subseteq W$.

Proof. Let $Z=\overline{\operatorname{co}}^{\tau}(\operatorname{rg} F)$. Take $A \in \mathscr{F}$. Then the vector measure $F_{A}$ : $(\Omega, \mathscr{F}) \rightarrow X$ satisfies $v=\Delta\left(F_{A}\right) \leq \Delta(F)$ and $F(A)=r(v)$. So, $F(A) \in K_{\Delta(F)}$. If $L=\left\{v \in M^{+}(X): 0 \leq v \leq \Delta(F)\right\}$, then $L$ is a compact convex set and the resultant map $r: M^{+}(X) \rightarrow \widehat{X}$ is linear and continuous when $\widehat{X}$ is endowed with the weak topology $\tau$ (see [C, vol. 3, page 6]); so we get that $K_{\Delta(F)}$ is a $\tau$-compact convex set and therefore $Z \subseteq K_{\Delta(F)}$.

Conversely, consider $v \in M^{+}(X), v \leq \Delta(F)$. Then for every $x^{*} \in X^{*}$, we have

$$
v\left(x^{*}\right) \leq v\left(\left(x^{*}\right)^{+}\right) \leq \Delta(F)\left(\left(x^{*}\right)^{+}\right)=\sup _{A \in \mathscr{F}} x^{*}(F(A))=\sup _{x \in Z} x^{*}(x),
$$

and an application of the Hahn-Banach theorem for the topology $\tau$ yields $K_{\Delta(F)} \subseteq Z$.

Last assertion in the theorem follows easily since the weak topology in $X$ is the restriction to $X$ of the $\tau$-topology and $\overline{\mathrm{co}}^{\tau}(\mathrm{rg} F)$ is $\tau$-compact due to the fact that $\operatorname{rg} F$ is bounded. $(\widehat{X}, \tau)$ is isomorphic to $\mathbb{R}^{I}$ for certain set $I$ and then every closed bounded set is compact.

As a consequence of the last theorem, if $X$ is a Banach space, $K_{\Delta(F)} \subseteq X$ if and only if $F$ is strongly additive. 
A result also due to Kluvánek asserts that every zonoform is the closed convex hull of the range of a vector measure; that is, given a conical measure $u$ in a locally convex space $X$ whose associated zonoform $K_{u}$ is contained in $X$, we can find an $X$-valued countably additive vector measure $F$ such that $u=\Delta(F)$ and, in particular, $K_{u}=\overline{\mathrm{co}}(\mathrm{rg} F)$. The proof of Kluvánek is purely existential and does not show any relationship between $u$ and $F$. Theorem 1.2 is devoted to give a new proof of this fact in the context of bounded measures. Our proof differs in some interesting details from the one in [K1] and [K2]; in particular, the measure $F$ is defined on a very natural algebra of sets of $X$ which only depends on $X$ and not on the conical measure $u$.

Let $X$ be a Hausdorff locally convex space. We define for every $x^{*} \in X^{*}$, the cones

$$
A_{x^{*}}=\left\{x \in X: x^{*}(x)>0\right\} \quad \text { and } C_{x^{*}}=\left\{x \in X: x^{*}(x) \geq 0\right\}
$$

Let $\mathscr{C}=\left\{A_{x^{*}}: x^{*} \in X^{*}\right\} \cup\left\{C_{x^{*}}: x^{*} \in X^{*}\right\}$ and $\mathscr{A} \mathscr{C}(X)$ be the algebra generated by the sets in $\mathscr{C}$. Then every element in $\mathscr{A} \mathscr{C}(X)$ can be written as

$$
A=\bigcup_{j \in J} H_{j},
$$

where $J$ is a finite set and $H_{j}$ is a finite intersection of elements in $\mathscr{C}$; that is,

$$
H_{j}=\left(\bigcap_{x^{*} \in I_{j}} A_{x^{*}}\right) \cap\left(\bigcap_{x^{*} \in K_{j}} C_{x^{*}}\right)
$$

for $I_{j}$ and $K_{j}$ finite sets in $X^{*}$. The sets in (3) are called elementary cones, that is, an elementary cone is a finite intersection of closed and open halfspaces. In addition, the sets $H_{j}$ in (2) can be chosen pairwise disjoint.

If $Z$ is now a linear subspace of $X^{*}$, we denote by $\mathscr{A} \mathscr{C}(X, Z)$ the algebra of subsets of $X$ generated by the family $\left\{A_{x^{*}}: x^{*} \in Z\right\} . \mathscr{A} \mathscr{C}(X, Z)$ is a subalgebra of $\mathscr{A} \mathscr{C}(X)$, and with this notation $\mathscr{A} \mathscr{C}(X)=\mathscr{A} \mathscr{C}\left(X, X^{*}\right)$. The elements of $\mathscr{A} \mathscr{C}(X, Z)$ are described in the same way as the elements of $\mathscr{A} \mathscr{C}(X)$ replacing $X^{*}$ by $Z$.

Our next aim is to define a finitely additive vector measure on $\mathscr{A} \mathscr{C}(X)$ whose associated conical measure is $u$.

THEOREM 1.2. Let $X$ be a Hausdorfflocally convex space. If $u$ is a conical measure on $X$ such that $K_{u} \subseteq X$, then there is a unique finitely additive bounded vector measure $\sigma(u): \mathscr{A} \mathscr{C}(X) \rightarrow X$ satisfying:

(a) The conical measure associated to $\sigma(u)$ is $u$.

(b) $\sigma(u)(H) \in H \cup\{0\}$ for every elementary cone $H$.

(c) For every finite dimensional subspace $Z$ of $X^{*}$, the restriction of $\sigma(u)$ to $\mathscr{A} \mathscr{C}(X, Z)$ is countably additive. 
To prove this theorem, we use several lemmas.

LEMMA 1.3. Let $u$ be a conical measure on $X$ such that $K_{u} \subseteq X$. Consider for every $x_{0}^{*} \in X^{*}$ the sets $A=\left\{x \in X: x_{0}^{*}(x)>0\right\}$ and $C=\left\{x \in X: x_{0}^{*}(x) \leq 0\right\}$. Then there exists a unique decomposition of $u$ in the form $u=u_{A}+u_{C}$, where $u_{A}$ and $u_{C}$ are conical measures such that if $v \in M^{+}(X)$ and $v \leq u_{A}, r(v) \in A \cup\{0\}$; and if $v \leq u_{C}, r(v) \in C$. In addition, if $v \leq u_{A}$ and $r(v)=0$, then $v=0$.

PROOF. For every $z^{*} \in h^{+}(X)$ and every $u \in M^{+}(X)$ we define $u_{A}\left(z^{*}\right)$ and $u_{C}\left(z^{*}\right)$ as

$$
\begin{aligned}
& u_{A}\left(z^{*}\right)=\lim _{n} u\left(\left(n x_{0}^{*} \wedge z^{*}\right)^{+}\right) \\
& u_{C}\left(z^{*}\right)=\left(u-u_{A}\right)\left(z^{*}\right)=\lim _{n} u\left(z^{*}-\left(n x_{0}^{*} \wedge z^{*}\right)^{+}\right) .
\end{aligned}
$$

It is easy to prove that $u_{A}$ and $u_{C}$ defined as above and linearly extended to $h(X)$ are conical measures. For example, the additivity of $u_{A}$ on $h^{+}(X)$ can be deduced from the following fact: if $z_{1}^{*}, z_{2}^{*} \in h^{+}(X)$, for every positive integer $n$ we have

$$
\left(n x_{0}^{*} \wedge\left(z_{1}^{*}+z_{2}^{*}\right)\right)^{+} \leq\left(n x_{0}^{*} \wedge z_{1}^{*}\right)^{+}+\left(n x_{0}^{*} \wedge z_{2}^{*}\right)^{+} \leq\left(2 n x_{0}^{*} \wedge\left(z_{1}^{*}+z_{2}^{*}\right)\right)^{+}
$$

which is easy to prove for real numbers and, consequently, for pointwise order of functions.

Observe first that $v \leq u_{C}$ implies $r(v) \in C$. Indeed, $x_{0}^{*}(r(v))=v\left(x_{0}^{*}\right)=$ $v\left(\left(x_{0}^{*}\right)^{+}\right)-v\left(\left(x_{0}^{*}\right)^{-}\right)$. Then it is enough to show that $v\left(\left(x_{0}^{*}\right)^{+}\right)=0$. Since $0 \leq$ $v\left(\left(x_{0}^{*}\right)^{+}\right) \leq u_{C}\left(\left(x_{0}^{*}\right)^{+}\right)$, we only have to show that $u_{C}\left(\left(x_{0}^{*}\right)^{+}\right)=0$. This is obvious since $\left(n x_{0}^{*} \wedge\left(x_{0}^{*}\right)^{+}\right)^{+}=\left(x_{0}^{*}\right)^{+}$and $u_{C}\left(\left(x_{0}^{*}\right)^{+}\right)=\lim _{n} u\left(\left(x_{0}^{*}\right)^{+}-\left(n x_{0}^{*} \wedge\left(x_{0}^{*}\right)^{+}\right)^{+}\right)=0$.

A similar argument shows that if $v \leq u_{A}$, then $x_{0}^{*}(r(v)) \geq 0$. We prove that, if in addition $x_{0}^{*}(r(v))=0$, then $v=0$ and consequently, $r(v)=0$. This means that $r(v) \in A \cup\{0\}$ for $v \leq u_{A}$.

We prove first that if $v \leq u_{A}$, then $v=v_{A}$. It is easy to prove that $\left(u_{A}\right)_{A}=u_{A}$. Let $z^{*} \in h^{+}(X)$, then

$$
\begin{aligned}
\left(u_{A}\right)_{A}\left(z^{*}\right) & =\lim _{m} u_{A}\left(\left(m x_{0}^{*} \wedge z^{*}\right)^{+}\right) \\
& =\lim _{m} \lim _{n} u\left(\left(n x_{0}^{*} \wedge\left(m x_{0}^{*} \wedge z^{*}\right)^{+}\right)^{+}\right)=\lim _{m} u\left(\left(m x_{0}^{*} \wedge z^{*}\right)^{+}\right),
\end{aligned}
$$

since $\left(n x_{0}^{*} \wedge\left(m x_{0}^{*} \wedge z^{*}\right)^{+}\right)^{+}=\left(m x_{0}^{*} \wedge z^{*}\right)^{+}$for every $n \geq m$. One must also observe that $\left(u_{A}-v\right)_{A} \leq u_{A}-v$, and $v_{A} \leq v$; but these inequalities must be equalities since $u_{A}=\left(u_{A}\right)_{A}=\left(\left(u_{A}-v\right)+v\right)_{A}=\left(u_{A}-v\right)_{A}+v_{A} \leq u_{A}-v+v=u_{A}$. We deduce that $v=v_{A}$.

By the definition of $u_{A}$, we have $u_{A}\left(\left(x_{0}^{*}\right)^{-}\right)=0$; therefore, $v\left(\left(x_{0}^{*}\right)^{-}\right)=0$, and $0=x_{0}^{*}(r(v))=v\left(x_{0}^{*}\right)=v\left(\left(x_{0}^{*}\right)^{+}\right)$. Thus $v\left(\left|x_{0}^{*}\right|\right)=v\left(\left(x_{0}^{*}\right)^{+}+\left(x_{0}^{*}\right)^{-}\right)=0$. This 
implies that $v=v_{A}=0$, since $\left(n x_{0}^{*} \wedge z^{*}\right)^{+} \leq n\left|x_{0}^{*}\right|$ for $z^{*} \in h^{+}(X)$, and we have $v_{A}\left(z^{*}\right)=0$. This implies that $v_{A}=0$, and $v=0$. This finishes the proof of the existence of $u_{A}$ and $u_{C}$ as in the statement of this lemma.

To finish the proof of Lemma 1.3, let us prove the uniqueness of $u_{A}$ and $u_{C}$. Suppose there exist two decompositions $u=u_{A}+u_{C}$ and $u=u_{A}^{\prime}+u_{C}^{\prime}$ satisfying $K_{u_{A}} \subseteq A \cup\{0\}, K_{u_{A}^{\prime}} \subseteq A \cup\{0\}, K_{u_{C}} \subseteq C$ and $K_{u_{C}^{\prime}} \subseteq C$. Consider the conical measure $w=u_{A} \vee u_{A}^{\prime}-u_{A} \wedge u_{A}^{\prime}$. Observe that $K_{w} \subseteq K_{u_{A} \vee u_{A}^{\prime}} \subseteq K_{u_{A}+u_{A}^{\prime}} \subseteq(A \cup\{0\})+(A \cup\{0\})=$ $A \cup\{0\}$. On the other hand, as $w=u_{C} \vee u_{C}^{\prime}-u_{C} \wedge u_{C}^{\prime}$, we deduce in the same way that $K_{w} \subseteq C$. So, we have that $K_{w}=\{0\}$. We are going to prove that this condition implies $w=0$. For every $x^{*} \in X^{*}$, as $r\left(w_{A_{x^{*}}}\right)=0$, we have that $w\left(\left(x^{*}\right)^{+}\right)=w_{A_{x^{*}}}\left(x^{*}\right)=x^{*}\left(r\left(w_{A_{x^{*}}}\right)\right)=0$. Using the same argument with $-x^{*}$, we conclude that $w\left(\left|x^{*}\right|\right)=0$. If $z^{*} \in h^{+}(X)$ can be written as $z^{*}=\bigvee_{i=1}^{n} x_{i}^{*}-\bigvee_{i=n+1}^{m} x_{i}^{*}$ and we have $\left|z^{*}\right| \leq \sum_{i=1}^{m}\left|x_{i}^{*}\right|$, then $w\left(z^{*}\right)=0$. Consequently, $w=0$. Then $u_{A} \vee u_{A}^{\prime}=u_{A} \wedge u_{A}^{\prime}$ and this means that $u_{A}=u_{A}^{\prime}, u_{C}=u_{C}^{\prime}$.

REMARK. It can be proved that $u_{C}$ coincides with the conical measure introduced in [C, Volume II, page 194] for every closed cone $C$ and defined for $z^{*} \in h^{+}(X)$ by

$$
u_{C}\left(z^{*}\right)=\inf \left\{u\left(w^{*}\right): w^{*} \in h^{+}(X), w^{*} \geq z^{*} \text { in } C\right\} .
$$

We do not make use of this fact, so we do not include its proof.

LEMMA 1.4. Let $u$ be a conical measure on $X$ such that $K_{u} \subseteq X$. For every elementary cone $H$ there exists a unique conical measure $u_{H}$ which satisfies

(a) $u_{H} \leq u$;

(b) $K_{u_{H}} \subseteq H \cup\{0\}$;

(c) for every conical measure $v$ with $v \leq u$ and $K_{v} \subseteq H \cup\{0\}$, we have $v \leq u_{H}$. If $0 \notin H$, we also have that $v \leq u_{H}$ and $r(v)=0$ implies $v=0$. If $H=\bigcup_{k=1}^{p} H_{k}$ is a partition of $H$ into elementary cones, then $u_{H}=\sum_{k=1}^{p} u_{H_{k}}$.

PROOF. The uniqueness of $u_{H}$ is obvious from condition (c). Every elementary cone is the intersection of a finite family of closed or open halfspaces; that is, for certain finite family $\left\{x_{1}^{*}, \ldots, x_{m}^{*}\right\}$ in $X^{*}$ and certain $n \in\{0,1, \ldots, m\}$ we can write

$$
H=\bigcap_{j=1}^{n}\left\{x: x_{j}^{*}(x) \leq 0\right\} \cap \bigcap_{j=n+1}^{m}\left\{x: x_{j}^{*}(x)>0\right\}=\bigcap_{j=1}^{n} C_{-x_{j}^{*}} \cap \bigcap_{j=n+1}^{m} A_{x_{j}^{*}} .
$$

In order to simplify the notation, we write $C_{j}^{+}=A_{x_{j}^{*}}$ and $C_{j}^{-}=C_{-x_{j}^{*}}$. If $\varepsilon \in\{+,-\}^{m}$ we denote by $H_{\varepsilon}=\bigcap_{j=1}^{m} C_{j}^{\varepsilon_{j}}$. Then $H=H_{\varepsilon^{0}}$ for certain $\varepsilon^{0} \in\{+,-\}^{m}$. Using Lemma 1.3, we define

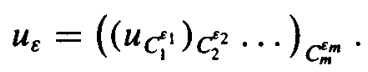


By the same lemma, we have that $\sum_{\varepsilon} u_{\varepsilon}=u$. Also, since $v \leq u$ this implies $v_{C} \leq u_{C}$, $v_{A} \leq u_{A}$, and we always have $u_{A} \leq u, u_{C} \leq u$; we deduce that $u_{\varepsilon} \leq u_{C_{j}^{i_{j}}}$ for every $j=1, \ldots, m$. We have then $K_{u_{\varepsilon}} \subseteq H_{\varepsilon} \cup\{0\}$ for every $\varepsilon$. If $0 \notin H_{\varepsilon}$, for some $j$, $\varepsilon_{j}=+$ and $C_{j}^{\varepsilon_{j}}=A$ is open. We have that if $v \leq u_{H_{\varepsilon}}, r(v)=0$ that $v \leq u_{A}$ and $r(v)=0$; by Lemma $1.3, v=0$.

If $H=H_{\varepsilon^{0}}$ and $u_{H}=u_{\varepsilon^{0}}$, let us prove that $u_{H}$ satisfies (c). Let $v \leq u$ be such that $K_{v} \subseteq H \cup\{0\}$. Using the Riesz decomposition, we have $v=\sum_{\varepsilon} v_{\varepsilon}$ with $v_{\varepsilon} \leq u_{\varepsilon}$ for every $\varepsilon$. If $\varepsilon \neq \varepsilon^{0}$, since $K_{v_{\varepsilon}} \subseteq K_{v}$, we have $K_{v_{\varepsilon}} \subseteq H \cup\{0\}$. On the other hand, $v_{\varepsilon} \leq u_{\varepsilon}$ and then $K_{v_{\varepsilon}} \subseteq H_{\varepsilon} \cup\{0\}$. Since $H \cap H_{\varepsilon}=\emptyset$, we have $K_{v_{\varepsilon}}=\{0\}$; which implies (see the proof of Lemma 1.3) that $v_{\varepsilon}=0$. We conclude that $v=v_{\varepsilon^{0}} \leq u_{H}$.

To finish the proof, if $H=\bigcup_{k=1}^{p} H_{k}$ is a partition of $H$ into disjoint elementary cones, we can choose a certain finite family $\left\{x_{1}^{*}, \ldots, x_{m}^{*}\right\}$ in $X^{*}$ (the functionals defining $H$ and every $H_{k}$ ) such that if, like before, we put $C_{j}^{+}=A_{x_{j}^{*}}, C_{j}^{-}=C_{-x_{j}^{*}}$ and $C_{\varepsilon}=\bigcap_{j=1}^{m} C_{j}^{\varepsilon_{j}}$ for every $\varepsilon \in\{+,-\}^{m}$ we have

$$
H=\bigcup_{C_{\varepsilon} \subseteq H} C_{\varepsilon} ; \quad H_{k}=\bigcup_{C_{\varepsilon} \subseteq H_{k}} C_{\varepsilon}, \quad k=1, \ldots, p .
$$

Following the argument to prove that $u_{H}$ satisfies (c), one can see that the conical measure $\sum_{C_{\varepsilon} \subseteq H} u_{C_{\varepsilon}}$ also satisfies (a), (b) and (c); so, $u_{H}=\sum_{C_{\varepsilon} \subseteq H} u_{C_{\varepsilon}}$ and analogously $u_{H_{k}}=\sum_{\mathcal{C}_{\varepsilon} \subseteq H_{k}} u_{C_{\varepsilon}}$, for $k=1, \ldots, p$. From this fact we deduce that $u_{H}=\sum_{k=1}^{p} u_{H_{k}}$.

REMARK. Suppose there exist a subset $S$ of $X$, a $\sigma$-algebra $\Sigma$ on $S$, and a positive measure $\mu$ in $\Sigma$ such that, for every $z^{*} \in h(X)$, its restriction to $S$ is measurable and $\mu$-integrable. Then $u\left(z^{*}\right)=\int_{S} z^{*} d \mu$, for every $z^{*} \in h(X)$, defines a conical measure on $X$. It is easy to see that for this conical measure and for every elementary cone $H$, the conical measure $u_{H}$ is given by

$$
u_{H}\left(z^{*}\right)=\int_{S \cap H} z^{*} d \mu .
$$

For, by the definition of $u_{H}$ made in the proof of Lemma 1.4, one is reduced to the case where $H$ is a closed or open halfspace. For a halfspace $H$ the above identity is an easy application of the dominated convergence theorem to the definitions given at the beginning of the proof of Lemma 1.3.

LEMMA 1.5. Suppose that $H$ is an elementary cone and $z_{1}^{*}, z_{2}^{*} \in h(X)$ satisfy $z_{1}^{*}=z_{2}^{*}$ on $H$. Then $u_{H}\left(z_{1}^{*}\right)=u_{H}\left(z_{2}^{*}\right)$.

PROOF. We can assume that $z_{1}^{*} \leq z_{2}^{*}$ since $z_{1}^{*} \wedge z_{2}^{*} \leq z_{i}^{*} \leq z_{1}^{*} \vee z_{2}^{*}$ for $i=1,2$ and all of them coincide on $H$. Then we only have to prove that $u_{H}\left(z^{*}\right)=0$ if $z^{*} \geq 0$ and 
$z^{*}=0$ on $H$. If $H \neq \emptyset$ and $H=\bigcap_{k=1}^{l}\left\{x: x_{k}^{*}(x)<0\right\} \cap \bigcap_{k=l+1}^{n}\left\{x: x_{k}^{*}(x) \leq 0\right\}$, then $\bar{H}=\bigcap_{k=1}^{n}\left\{x: x_{k}^{*}(x) \leq 0\right\}$. If we prove our assertion for $\bar{H}$, it will also be true for $H$ since $u_{\bar{H}} \geq u_{H}$.

So we assume that $H=\bigcap_{k=1}^{n} C_{k}$, where $C_{k}=\left\{x: x_{k}^{*}(x) \leq 0\right\}$. We prove that under this condition, if $z^{*} \in h(X)$ and $z^{*} \leq 0$ on $H$, then there exists a constant $\alpha>0$ such that

$$
z^{*}(x) \leq \alpha \sum_{k=1}^{n}\left(x_{k}^{*}\right)^{+}(x), \quad \text { for every } x \in X .
$$

Once (4) is proved, if $z^{*}=0$ on $H$ and $z^{*} \geq 0$, we would have

$$
0 \leq u_{H}\left(z^{*}\right) \leq \alpha \sum_{k=1}^{n} u_{H}\left(\left(x_{k}^{*}\right)^{+}\right) \leq \alpha \sum_{k=1}^{n} u_{C_{k}}\left(\left(x_{k}^{*}\right)^{+}\right)=0
$$

and the proof would be finished.

To prove (4), suppose that $z^{*}=\bigvee_{i=1}^{l} a_{i}^{*}-\bigvee_{j=1}^{J} b_{j}^{*}$, where each $a_{i}^{*} \in X^{*}, b_{j}^{*} \in X^{*}$ and $I, J$ are positive integers. Then

$$
z^{*}=\bigvee_{i=1}^{I}\left(a_{i}^{*}-\bigvee_{j=1}^{J} b_{j}^{*}\right)=\bigvee_{i=1}^{I}\left(\bigwedge_{j=1}^{J}\left(a_{i}^{*}-b_{j}^{*}\right)\right)=\bigvee_{i=1}^{I} z_{i}^{*}
$$

If $z^{*} \leq 0$ on $H$, then each $z_{i}^{*}=\bigwedge_{j=1}^{J}\left(a_{i}^{*}-b_{j}^{*}\right) \leq 0$ on $H$ and it is enough to prove (4) for $z_{i}^{*}$. So we may assume without loss of generality that $z^{*}=\bigwedge_{j=1}^{J} y_{j}^{*}$ with $y_{j}^{*} \in X^{*}$. We assume that the following claim is true and we postpone its proof.

CLAIM. If $H=\bigcap_{k=1}^{n}\left\{x: x_{k}^{*}(x) \leq 0\right\}$ and $H \subseteq\left\{x \in X: y^{*}(x) \leq 0\right\}$ for some $y^{*} \in X^{*}$, then $y^{*} \in L$, where $L$ is the set defined by

$$
L=\left\{\sum_{k=1}^{n} \alpha_{k} x_{k}^{*}: \alpha_{k} \geq 0, \quad k=1, \ldots, n\right\}
$$

The fact that $z^{*}=\bigwedge_{j=1}^{J} y_{j}^{*} \leq 0$ on $H$ means that $H \cap G=\emptyset$, where $G$ is the open cone $G=\bigcap_{j=1}^{J}\left\{x: y_{j}^{*}(x)>0\right\}$. If $G=\emptyset$, there is nothing to prove. Otherwise, $G$ is a non-empty open cone disjoint from $H$. Then, by the Hahn-Banach theorem, there is $y^{*} \in X^{*}$ such that $G \subseteq\left\{x \in X: y^{*}(x)>0\right\}$ and $H \subseteq\left\{x \in X: y^{*}(x) \leq 0\right\}$. Therefore, by application of our claim let us find $\alpha_{k} \geq 0$ with $y^{*}=\sum_{k=1}^{n} \alpha_{k} x_{k}^{*}$ and $\beta_{j} \geq 0$ with $y^{*}=\sum_{j=1}^{J} \beta_{j} y_{j}^{*}$. Since $y^{*} \neq 0$, there exists $j_{0}$ such that $\beta_{j_{0}}>0$. Put $\alpha=\left(\max _{1 \leq k \leq n} \alpha_{k}\right) / \beta_{j_{0}}$. Then, if $x \in X$ satisfies $z^{*}(x) \leq 0$, there is nothing to prove in (4); if $z^{*}(x)>0$, then $y_{j}^{*}(x)>0$ for every $j=1, \ldots, n$ and

$$
z^{*}(x)=\bigwedge_{j=1}^{J} y_{j}^{*}(x) \leq y_{j_{0}}^{*}(x) \leq \frac{1}{\beta_{j_{0}}} \sum_{j=1}^{J} \beta_{j} y_{j}^{*}(x)=\frac{1}{\beta_{j_{0}}} \sum_{k=1}^{n} \alpha_{k} x_{k}^{*}(x) \leq \alpha \sum_{k=1}^{n}\left(x_{k}^{*}\right)^{+}(x) .
$$

This concludes the proof of (4) and the proof of Lemma 1.5. 
Proof OF THE Claim. We first observe that $y^{*} \in C$, where $C$ is the $\sigma\left(X^{*}, X\right)$ closed cone generated by $x_{k}^{*}, k=1, \ldots, n$. If this is not true, an application of the Hahn-Banach theorem, let us find an element $x_{0} \in X$ such that $y^{*}\left(x_{0}\right)>0$ and $x_{k}^{*}\left(x_{0}\right) \leq 0$ for $k=1, \ldots, n$. So $x_{0} \in H$ but $x_{0} \notin\left\{x \in X: y^{*}(x) \leq 0\right\}$ and this contradicts our hypothesis. This shows that $y^{*} \in C$.

We next observe that $L$ is a closed set for $\sigma\left(X^{*}, X\right)$. This fact can be easily proved by induction on $n$. For $n=1$, it is obvious. Assuming this is true for $n-1$, we prove that $L$, defined as in (5), is closed. For $s \in\{1, \ldots, n\}$ fixed, we define the set $L_{s}$ by

$$
L_{s}=\left\{\sum_{k=1}^{n} \alpha_{k} x_{j}^{*}: \alpha_{k} \geq 0, \quad j=1, \ldots, n ; \alpha_{s}=0\right\} .
$$

So, each set $L_{s}$ is closed by our induction hypothesis. Let $K$ be the convex hull of the set $\left\{x_{1}^{*}, \ldots, x_{n}^{*}\right\}$. If $0 \in K$, then $0=\sum_{k=1}^{n} \alpha_{k} x_{k}^{*}$ where $\sum_{k=1}^{n} \alpha_{k}=1$ and each $\alpha_{k} \geq 0$. If $x=\sum_{k=1}^{n} \beta_{k} x_{k}^{*}$ is an element of $L$ with every $\beta_{k} \geq 0$, then either there exists $s \in\{1, \ldots, n\}$ with $\beta_{s}=0$ and $x \in L_{s}$ or we have $\beta_{k}>0$, for each $k$. In this latter case, taking $\lambda$ small enough, we obtain that $x=\sum_{k=1}^{n}\left(\beta_{k}-\lambda \alpha_{k}\right) x_{k}^{*}$ where for each $k, \beta_{k}-\lambda \alpha_{k} \geq 0$ and for some $s, \beta_{s}-\lambda \alpha_{s}=0$. Then $x \in L_{s}$ in this case, too. So, if $0 \in K, L=\bigcup_{s=1}^{n} L_{s}$ which proves that $L$ is closed in this case.

If $0 \notin K$, we observe first that the closed linear span of $L$ is finite dimensional, so we can choose a norm in this space to define its topology. So there exists $\varepsilon>0$ such that the ball $B(0, \varepsilon)$ for this norm does not intersect the compact set $K$. Let $w^{*} \in \bar{L}$ and $\left(w_{p}^{*}\right)_{p}$ be a sequence contained in $L$ converging to $w^{*}$, with $w_{p}^{*}=\sum_{k=1}^{n} \alpha_{k, p} x_{k}^{*}$, $\alpha_{k, p} \geq 0$. Then there exists a constant $M>0$ such that $\left(w_{p}^{*}\right)_{p} \subseteq B(0, M \varepsilon)$. Assume that there exists $p$ such that $\gamma=\sum_{k=1}^{n} \alpha_{k, p}>M$, then $w_{p}^{*} / \gamma \in K \cap B(0, \varepsilon)$, which is a contradiction. So $\sum_{k=1}^{n} \alpha_{k, p} \leq M$, for every $p$, and this implies, by a compactness argument, that $w^{*}=\sum_{k=1}^{n} \alpha_{k} x_{k}^{*}$, with $\alpha_{k} \geq 0$, and, in particular, $w^{*} \in L$. This shows that $L$ is closed, $L=C$ and the claim follows.

The proof of Lemma 1.5 could have been given using localization of conical measures in finite dimension and observing that, in this case, if $\mu$ is a localization of $u$, the restriction of $\mu$ to $H$ is a localization of $u_{H}$.

ProOF OF THEOREM 1.2. Let $u$ be a conical measure on $X$ such that $K_{u} \subseteq X$. We define for every elementary cone $H$ in $X, \sigma(u)(H)=r\left(u_{H}\right)$. The additivity of the resultant and Lemma 1.4 let us extend $\sigma(u)$ to $\mathscr{A} \mathscr{C}(X)$, the algebra generated by the elementary cones. Then $\sigma(u)$ is a finitely additive vector measure whose range is contained in $K_{u}$ and such that $\sigma(u)(H) \in H \cup\{0\}$ for every elementary cone $H$.

Let $\tilde{u}$ be the conical measure associated to $\sigma(u)$. We have to show that $u=\tilde{u}$. Let $z^{*} \in h(X), z^{*}=\bigvee_{i=1}^{n} x_{i}^{*}$. There exists a partition of $X$ into elementary cones 
$X=\bigcup_{j=1}^{n} H_{j}$ in such a way that $z^{*}=x_{j}^{*}$ in $H_{j}$ for $j=1, \ldots, n$. It is enough to take

$$
H_{j}=\left\{x \in X:\left(x_{j}^{*}-x_{k}^{*}\right)(x) \geq 0, \text { for } j \leq k,\left(x_{j}^{*}-x_{k}^{*}\right)(x)>0 \text {, for } k<j\right\} .
$$

By the definition of $\sigma(u)$, we have for every $A \in \mathscr{A} \mathscr{C}(X)$ with $A \subseteq H_{j}, \sigma(u)(A) \in$ $H_{j} \cup\{0\}$. This tells us that for $k \neq j$, the measure $\left(x_{j}^{*}-x_{k}^{*}\right) \circ \sigma(u)$ is positive on the sets of $\mathscr{A} \mathscr{C}(X)$ contained in $H_{j}$. Therefore, $\Phi_{\sigma(u)}\left(z^{*}\right)(A)=x_{j}^{*}(\sigma(u)(A))$ for every $A \subseteq H_{j}$ and

$$
\begin{aligned}
\tilde{u}\left(z^{*}\right) & =\Phi_{\sigma(u)}\left(z^{*}\right)(X)=\sum_{j=1}^{n} \Phi_{\sigma(u)}\left(z^{*}\right)\left(H_{j}\right)=\sum_{j=1}^{n} x_{j}^{*}\left(\sigma(u)\left(H_{j}\right)\right) \\
& =\sum_{j=1}^{n} x_{j}^{*}\left(r\left(u_{H_{j}}\right)\right)=\sum_{j=1}^{n} u_{H_{j}}\left(x_{j}^{*}\right)=\sum_{j=1}^{n} u_{H_{j}}\left(z^{*}\right)=u\left(z^{*}\right),
\end{aligned}
$$

last two equalities thanks to Lemma 1.5 and Lemma 1.4, respectively.

We postpone the proof that $\sigma(u)$ satisfies the condition (c) of the statement of this theorem to the next section. In fact, we prove there that, when $X$ is a complete weak space, then $\sigma(u)$ is countably additive on the whole algebra $\mathscr{A} \mathscr{C}(X)$. We deduce the condition (c) from this fact in Corollary 2.5 in order to avoid repeating the same arguments in both sections.

In order to prove the uniqueness of $\sigma(u)$, suppose that $F: \mathscr{A} \mathscr{C}(X) \rightarrow X$ is a finitely additive bounded vector measure satisfying the same conditions. We are going to see that then, for every elementary cone $H$, the conical measure $\Delta\left(F_{H}\right)$ associated to $F_{H}$ is the conical measure $u_{H}$ (remember that $F_{H}$ is defined by $F_{H}(A)=F(A \cap H)$ for every $A \in \mathscr{A} \mathscr{C}(X))$. Then we have, by the definition of $\sigma(u)$,

$$
\sigma(u)(H)=r\left(u_{H}\right)=r\left(\Delta\left(F_{H}\right)\right)=F_{H}(X)=F(H),
$$

and therefore $F=\sigma(u)$.

It is enough to prove $\Delta\left(F_{H}\right) \leq u_{H}$ for every elementary cone $H$. For given $H$, we can construct a finite partition $\left\{H_{0}, H_{1}, \ldots, H_{n}\right\}$ of $X$ into elementary cones such that $H=H_{0}$. Then we would have

$$
\Delta\left(F_{H}\right)+\sum_{j=1}^{n} \Delta\left(F_{H_{j}}\right)=\Delta(F)=u=u_{H}+\sum_{j=1}^{n} u_{H_{j}},
$$

thus we get the other inequality since

$$
\Delta\left(F_{H}\right)=u_{H}+\sum_{j=1}^{n}\left(u_{H_{j}}-\Delta\left(F_{H_{j}}\right)\right) \geq u_{H} .
$$

Using Lemma 1.4, being $\Delta\left(F_{H}\right) \leq u$, we only have to prove that the zonoform $K_{\Delta\left(F_{H}\right)}=\overline{\mathrm{co}}\left(\mathrm{rg} F_{H}\right)$ is contained in $H \cup\{0\}$ to get $\Delta\left(F_{H}\right) \leq u_{H}$. It suffices to prove 
$\overline{\mathrm{co}}\left(\mathrm{rg} F_{H}\right) \subset H \cup\{0\}$ when $H$ is a halfspace, since every elementary cone is a finite intersection of halfspaces. The condition $F(H) \in H \cup\{0\}$, for every elementary cone $H$, easily implies that $\operatorname{rg}\left(F_{H}\right) \subset H \cup\{0\}$ for every elementary cone $H$, and that $\overline{\mathrm{co}}\left(\mathrm{rg} F_{H}\right) \subset H$ when $H$ is closed.

The problem is reduced to proving $\overline{\operatorname{co}}\left(\operatorname{rg} F_{A}\right) \subset A \cup\{0\}$ when $A$ is the open halfspace $A=\left\{x \in X: x^{*}(x)>0\right\}$, for certain $x^{*} \in X^{*}$. Suppose $y \in \overline{\mathrm{co}}\left(\mathrm{rg} F_{A}\right)$, $y \neq 0$, and $x^{*}(y) \leq 0$. Clearly we have $x^{*}(y)=0$, and there exists $y^{*} \in X^{*}$ such that $y^{*}(y)=1$. For every $n \in \mathbb{N}$, let $y_{n}^{*}=y^{*}-n x^{*}, E_{n}=\left\{x \in A: y_{n}^{*}(x)>0\right\}$, and $C_{n}=\left\{x \in A: y_{n}^{*}(x) \leq 0\right\}$. The sets $E_{n}$ and $C_{n}$ belong to $\mathscr{A} \mathscr{C}(X, Z)$ where $Z$ is the vector space spanned by $x^{*}$ and $y^{*} ;$ as $\bigcap_{n} E_{n}=\emptyset$ we have $\lim _{n} y^{*}\left(F\left(E_{n}\right)\right)=0$. We can choose $m$ such that $y^{*}\left(F\left(E_{m}\right)\right) \leq 1 / 2$. Observe that $y^{*} \circ F$ and $x^{*} \circ F$ are positive measures inside $E_{m}$ and $y_{m}^{*} \circ F$ is negative inside $C_{m}$. Then we have, for every $M \in \mathscr{A} \mathscr{C}(X)$,

$$
\begin{aligned}
1 / 2 & \geq y^{*}\left(F\left(E_{m}\right)\right) \geq y^{*}\left(F\left(E_{m} \cap M\right)\right) \geq y_{m}^{*}\left(F\left(E_{m} \cap M\right)\right) \\
& \geq y_{m}^{*}\left(F\left(E_{m} \cap M\right)\right)+y_{m}^{*}\left(F\left(C_{m} \cap M\right)\right)=y_{m}^{*}(F(A \cap M)),
\end{aligned}
$$

which is a contradiction since $y \in \overline{\mathrm{co}}\left(\operatorname{rg} F_{A}\right), y_{m}^{*}(y)=1$ and $y_{m}^{*}(x) \leq 1 / 2$, for every $x \in \operatorname{rg} F_{A}$. This concludes the proof of Theorem 1.2.

We have used condition $(c)$ in order to obtain the uniqueness of $\sigma(u)$. This is necessary, even for a finite dimensional space $X$, as the following example shows.

EXAMPLE 1.6. There exist two finitely additive bounded measures $F$ and $G$ defined on $\mathscr{A} \mathscr{C}\left(\mathbb{R}^{2}\right)$ with values in $\mathbb{R}^{2}$ such that $F(H), G(H) \in H \cup\{0\}$ for every elementary cone $H, \Delta(F)=\Delta(G)$, but $F \neq G$.

PROOF. Let $e$ be the vector in $\mathbb{R}^{2}, e=(1,0)$. For $A \in \mathscr{A} \mathscr{C}\left(\mathbb{R}^{2}\right)$, define $F_{1}(A)=e$, if $e \in A$, and $F_{1}(A)=0$, if $e \notin A$; and define $G_{1}(A)=e$, if $e$ is an accumulation point of $A \cap\left\{(x, y) \in \mathbb{R}^{2}: y>0\right\}$, and $G_{1}(A)=0$, if not. Observe that, being the elements of $\mathscr{A} \mathscr{C}\left(\mathbb{R}^{2}\right)$ finite unions of angles, $G_{1}(A)=e$ if and only if $A$ contain some open angle determined by the positive $x$-axis and a half-line contained in the upper half-space $\left\{(x, y) \in \mathbb{R}^{2}: y>0\right\}$. Then it is easy to see that $G_{1}$ and $F_{1}$ are bounded vector measures having the same associated conical measure. $F_{1}$ is countably additive, but $G_{1}$ is not.

$G_{1}$ satisfies $G_{1}(H) \in H \cup\{0\}$ for every closed elementary cone $H$, but not for every elementary cone. In order to achieve this condition we have to perturb $G_{1}$. We will make the same perturbation to $F_{1}$. Let $C$ be the unit circle $C=\left\{(x, y) \in \mathbb{R}^{2}\right.$ : $\left.x^{2}+y^{2}=1\right\}$ and consider there the arc-length measure $m$. Define

$$
F_{0}(A)=\int_{A \cap C}(x, y) d m(x, y), \quad A \in \mathscr{A} \mathscr{C}\left(\mathbb{R}^{2}\right) .
$$


Observe that $F_{0}(H) \in H$ for every open elementary cone $H$. Then it is easy to see that $F=F_{0}+F_{1}$, and $G=F_{0}+G_{1}$ satisfy the required properties. In particular the conical measure $u$ associated to them is given by $u\left(z^{*}\right)=\int_{C} z^{*} d m+z^{*}(e)$, for every $z^{*} \in h\left(\mathbb{R}^{2}\right)$.

To finish this section, we collect some properties of the conical measure $u_{C}$ to be used later.

PROPOSITION 1.7. Let $u, v$ be conical measures on $X$ such that $K_{u}, K_{v} \subseteq X$ and let $C, C^{\prime}$ be elementary cones. Then

(a) $(u+v)_{C}=u_{C}+v_{C}$.

(b) $(u \wedge v)_{C}=u_{C} \wedge v=u_{C} \wedge v_{C}$.

(c) $u_{C} \wedge u_{C^{\prime}}=\left(u_{C^{\prime}}\right)_{C}=u_{C \cap C^{\prime}}$.

(d) If $\Gamma$ is a subset of $M^{+}(X)$, such that $K_{u} \subseteq X$ for every $u \in \Gamma$ and inf $\Gamma=v$, then $v_{C}=\inf \left\{u_{C}: u \in \Gamma\right\}$.

Proof. (a) easily follows from the definition of $u_{C}$ and $v_{C}$. To prove (b), let $w=u_{C} \wedge v$. As $w \leq u_{C}$, we have $w=w_{C}$ since $K_{w} \subseteq C \cup\{0\}$. As $w \leq u$ and $w \leq v$, we get $w \leq(u \wedge v)$ and $w=w_{C} \leq(u \wedge v)_{C}$. On the other hand, $(u \wedge v)_{C} \leq u_{C}$ and $(u \wedge v)_{C} \leq v_{C}$, therefore $(u \wedge v)_{C} \leq w$.

As a consequence of (b), with $v=u_{C^{\prime}}$, we have $u_{C} \wedge u_{C^{\prime}}=\left(u \wedge u_{C^{\prime}}\right)_{C}=\left(u_{C^{\prime}}\right)_{C}$. The second equality of (c) follows easily from the definition in the proof of Lemma 1.4. Let us show (d) now. If $w=\inf \left\{u_{C}: u \in \Gamma\right\}$, then $w \leq u$ for every $u \in \Gamma$. So, $w \leq v$. Since $w \leq u_{C}$ for some $u \in \Gamma, w=w_{C} \leq v_{C}$. As $v \leq u$ for every $u \in \Gamma$, $v_{C} \leq u_{C}$ for every $u \in \Gamma$. So $v_{C} \leq w$.

\section{Conical measures on complete weak spaces}

The bounded vector measure $\sigma(u)$ associated in Theorem 1.2 to the conical measure $u$ is not necessarily countably additive (see Section 3 for examples in the Banach space setting). However, in this section we prove that if $X$ is a complete weak space, $\sigma(u)$ is always countably additive. This allows us to obtain a new proof of two results due to Kluvánek: Corollary 2.4 and Theorem 2.8 , and to complete the proof of Theorem 1.2 in Corollary 2.5 .

If $X$ is a complete weak space then there exists a set $I$ such that, up to a topological vector isomorphism, $X=\mathbb{R}^{I}$. For every $\alpha \in I$, let $e_{\alpha}^{*}$ be the projection of $X$ onto its $\alpha$-th coordinate; that is, $e_{\alpha}^{*}\left(\left(x_{i}\right)_{i \in I}\right)=x_{\alpha}$ for every $\left(x_{i}\right)_{i \in l} \in X$. Every $x^{*} \in X^{*}$ is then a (finite) linear combination of the $e_{\alpha}^{*}$ 's, and so, every $z^{*} \in h(X)$, and every $A \in \mathscr{A} \mathscr{C}(X)$ depends upon a finite number of coordinates. Let us suppose that $I$ is 
the disjoint union of $\mathbb{N}$ and another set $I^{\prime}$. Then we can write $X=\mathbb{R}^{\prime}=\mathbb{R}^{\mathbb{N}} \times \mathbb{R}^{I^{\prime}}$. With this notation, we can prove the following lemma.

LEMMA 2.1. Let $u$ be a conical measure on $X=\mathbb{R}^{\prime}=\mathbb{R}^{N} \times \mathbb{R}^{I^{\prime}}$. If $\varepsilon>0, e^{*} \in X^{*}$ and $C=\left\{x \in X: e^{*}(x)>0\right\}$, there exists a sequence of positive numbers $\left\{M_{j}\right\}_{j \in \mathbb{N}}$ with the following property: for every $A \in \mathscr{A} \mathscr{C}(X)$ such that $e^{*}(\sigma(u)(A \cap C)) \geq \varepsilon$ we have $A \cap C \cap K \neq \emptyset$ where $K=\bigcap_{j=1}^{\infty}\left\{x \in X:\left|e_{j}^{*}(x)\right| \leq M_{j} e^{*}(x)\right\}$.

PROOF. Let us remark that, inside $C, e^{*} \circ \sigma(u)$ is a finitely additive positive measure. We prove first that if $e^{*}(\sigma(u)(C))>\alpha$ for some $\alpha \in \mathbb{R}$, then there exists $M_{j}$ such that $e^{*}\left(\sigma(u)\left(C \cap\left\{x \in X:\left|e_{j}^{*}(x)\right| \leq M_{j} e^{*}(x)\right\}\right)\right)>\alpha$. This will be clear once we have proved that, for every conical measure $u$ and $j$ fixed,

$$
\lim _{k \rightarrow \infty} e^{*}\left(\sigma(u)\left(C \cap\left\{x \in X:\left|e_{j}^{*}(x)\right| \leq k e^{*}(x)\right\}\right)\right)=e^{*}(\sigma(u)(C)) .
$$

Let $C_{k}=\left\{x \in X:\left|e_{j}^{*}(x)\right| \leq k e^{*}(x)\right\}, x_{k}=\sigma(u)\left(C \cap\left\{x \in X: e_{j}^{*}(x)>k e^{*}(x)\right\}\right)$ and $y_{k}=\sigma(u)\left(C \cap\left\{x \in X: e_{j}^{*}(x)<-k e^{*}(x)\right\}\right)$. Since $e^{*}(\sigma(u)(C))=e^{*}(\sigma(u)(C \cap$ $\left.\left.C_{k}\right)\right)+e^{*}\left(x_{k}\right)+e^{*}\left(y_{k}\right)$, it is enough to show that $e^{*}\left(x_{k}\right) \rightarrow 0$ and $e^{*}\left(y_{k}\right) \rightarrow 0$ as $k \rightarrow \infty$. If $e^{*}\left(x_{k}\right)$ does not converges to 0 as $k \rightarrow \infty$, there exists $\delta>0$ such that $e^{*}\left(x_{k}\right) \geq \delta>0$, for any $k$. By the properties of $\sigma(u), x_{k} \in C \cap\left\{x \in X: e_{j}^{*}(x)>\right.$ $\left.k e^{*}(x)\right\}$, so $e_{j}^{*}\left(x_{k}\right)>k \delta$ which implies that $e_{j}^{*}$ is not bounded on $K_{u}$, a contradiction. In the same way we can prove that $e^{*}\left(y_{k}\right) \rightarrow 0$ as $k \rightarrow \infty$.

Now we prove, by induction on $j$, that for any $\varepsilon>0$ there exists a sequence of positive numbers $\left\{M_{j}\right\}_{j}$ such that

$$
e^{*}\left(\sigma(u)\left(C \cap\left(\bigcap_{k=1}^{j}\left\{x \in X:\left|e_{k}^{*}(x)\right| \leq M_{k} e^{*}(x)\right\}\right)\right)\right)>e^{*}(\sigma(u)(C))-\varepsilon
$$

for every $j \in \mathbb{N}$. Assume that we have chosen $M_{1}, \ldots, M_{j}$. An application of the result of the previous paragraph to $u_{H_{j}}$, where $H_{j}=\bigcap_{k=1}^{\prime}\left\{x \in X:\left|e_{k}^{*}(x)\right| \leq\right.$ $\left.M_{k} e^{*}(x)\right\}$, lets us find a positive number $M_{j+1}$ such that the condition

$$
e^{*}\left(\sigma(u)\left(C \cap\left(\bigcap_{k=1}^{j+1}\left\{x \in X:\left|e_{k}^{*}(x)\right| \leq M_{k} e^{*}\right\}\right)\right)\right)>e^{*}(\sigma(u)(C))-\varepsilon
$$

is still satisfied. Observe that we have used that $\sigma\left(u_{A}\right)(B)=\sigma(u)(A \cap B)$ for $A$ and $B$ elementary cones.

Let $K$ be the set $K=\bigcap_{j=1}^{\infty}\left\{x \in X:\left|e_{j}^{*}(x)\right| \leq M_{j} e^{*}(x)\right\}=\bigcap_{j=1}^{\infty} H_{j}$. Suppose that $A \in \mathscr{A} \mathscr{C}(X)$ satisfies $e^{*}(\sigma(u)(A \cap C)) \geq \varepsilon$. Then $e^{*}\left(\sigma(u)\left(A \cap H_{j}\right)\right)>0$ for every $j$. We choose $j_{0}$ large enough such that $A \cap C$ is independent of the coordinates $j>j_{0}, j \in \mathbb{N}$. There is a point $a \in A \cap C \cap H_{j_{0}}$. Taking a point $b$ such that $e_{j}^{*}(a)=e_{j}^{*}(b)$ for $j \leq j_{0}, e_{j}^{*}(b)=0$ for $j>j_{0}, j \in \mathbb{N}$ and $e_{i}^{*}(a)=e_{i}^{*}(b)$ for $i \in I^{\prime}$, we have $b \in A \cap C \cap K$ and $A \cap C \cap K \neq \emptyset$. 
LEMMA 2.2. Let $X$ be a locally convex Hausdorff space and $u$ a conical measure on $X$ such that $K_{u} \subseteq X$. If $\varepsilon>0, e^{*} \in X^{*}, C=\left\{x \in X: e^{*}(x)>0\right\}$, and $A \in \mathscr{A} \mathscr{C}(X)$, then there exists $A^{\prime} \in \mathscr{A} \mathscr{C}(X), A^{\prime}$ closed, such that $C \cap A^{\prime} \subseteq C \cap A$ and $\varepsilon+e^{*}\left(\sigma(u)\left(C \cap A^{\prime}\right)\right) \geq e^{*}(\sigma(u)(C \cap A))$.

PROOF. Let $y^{*} \in X^{*}$ and $H=\left\{x \in X: e^{*}(x)>0, y^{*}(x)>0\right\}$. If $r>0$ we take $H_{r}=H_{r}\left(y^{*}\right)=\left\{x \in X: e^{*}(x)>0, y^{*}(x)>0, y^{*}(x)<r e^{*}(x)\right\}$. We prove that $e^{*}\left(\sigma(u)\left(H_{r}\right)\right) \rightarrow 0$ as $r \rightarrow 0^{+}$. If this is not true, there exists $\varepsilon>0$ such that $e^{*}\left(\sigma(u)\left(H_{r}\right)\right) \geq \varepsilon$, for $r>0$. Then $y_{r}=\sigma(u)\left(H_{r}\right) \in K_{u_{H_{r}}} \subseteq K_{u_{H}}$ which is a weakly compact set. Let $y$ be any weak accumulation point of the net $\left(y_{r}\right)_{r}$. Therefore, $e^{*}(y) \geq \varepsilon>0$. On the other hand, $y^{*}\left(y_{r}\right) \leq r e^{*}\left(y_{r}\right) \leq r \sup _{x \in K_{u}} e^{*}(x)$ and this implies that $y^{*}\left(y_{r}\right) \rightarrow 0$ as $r \rightarrow 0^{+}$. Then $y \in\left\{x \in X: y^{*}(x)=0\right\} \cap K_{u_{H}}=\{0\}$, a contradiction since $e^{*}(y) \neq 0$.

Next we observe that it is enough to prove this lemma for $A$ an elementary cone since an arbitrary $A \in \mathscr{A} \mathscr{C}(X)$ is a disjoint union of a finite family of elementary cones. So we may assume that

$$
A=\bigcap_{j \in l}\left\{x: x_{j}^{*}(x)>0\right\} \cap \bigcap_{j \in J}\left\{x: x_{j}^{*}(x) \geq 0\right\},
$$

where $I$ and $J$ are finite sets. Taking

$$
A^{\prime}=\bigcap_{j \in I}\left\{x: x_{j}^{*}(x) \geq r e^{*}(x)\right\} \cap \bigcap_{j \in J}\left\{x: x_{j}^{*}(x) \geq 0\right\}
$$

for $r>0$ small enough, we obtain

$$
\varepsilon+e^{*}\left(\sigma(u)\left(C \cap A^{\prime}\right)\right) \geq e^{*}(\sigma(u)(C \cap A))
$$

since $(A \cap C) \backslash\left(A^{\prime} \cap C\right) \subseteq \bigcup_{j \in l} H_{r}\left(x_{j}^{*}\right)$.

THEOREM 2.3. Let $X$ be a complete weak space. If $u$ is a conical measure on $X$ then $\sigma(u): \mathscr{A} \mathscr{C}(X) \rightarrow X$ is countably additive.

ProOF. First of all, observe that if $X$ is a complete weak space, the condition in Theorem 1.2 is fulfilled, that is, $K_{u} \subseteq X$. We only have to show that given a sequence $\left\{A_{n}\right\}$ of sets in $\mathscr{A} \mathscr{C}(X)$ that decreases to the empty set, then $\sigma(u)\left(A_{n}\right)$ converges to 0 . Keep in mind that every complete weak space $X$ is isomorphic to $\mathbb{R}^{I}$ for some set $I$. We proceed by a way of contradiction. If $\sigma(u)\left(A_{n}\right)$ does not converges to 0 , there is a coordinate functional $e^{*}$ such that $e^{*}\left(\sigma(u)\left(A_{n}\right)\right)=u_{A_{n}}\left(e^{*}\right)$ does not converges to 0 . Then either $u_{A_{n}}\left(\left(e^{*}\right)^{+}\right)$does not converge to 0 or $u_{A_{n}}\left(\left(e^{*}\right)^{-}\right)$ does not converge to 0 . We can assume that $u_{A_{n}}\left(\left(e^{*}\right)^{+}\right)=e^{*}\left(\sigma(u)\left(A_{n} \cap C\right)\right)$ does not converge to 0 , where $C=\left\{x \in X: e^{*}(x)>0\right\}$. Then there is a number $\varepsilon>0$ such that $e^{*}\left(\sigma(u)\left(A_{n} \cap C\right)\right)>\varepsilon$ for every $n$. 
Let us suppose that $A_{n}=\bigcup_{k \in K_{n}} C_{k}$ where each $C_{k}$ is an elementary cone and $K_{n}$ is a finite set for every $n$. If $C_{k}=\bigcap_{j \in I_{k}}\left\{x: x_{j}^{*}(x)>0\right\} \cap \bigcap_{j \in J_{k}}\left\{x: x_{j}^{*}(x) \geq 0\right\}$, where $I_{k}$ and $J_{k}$ are finite sets, the collection $\left\{x_{j}^{*}: j \in I_{k} \cup J_{k}, k \in \mathbb{N}\right\} \cup\left\{e^{*}\right\}$ is countable, so it spans a countable dimensioned subspace of $X^{*}$. Then we can write $\mathbb{R}^{I}=\mathbb{R}^{\mathbb{N}} \times \mathbb{R}^{I^{\prime}}$ where each $e^{*}, x_{j}^{*} \in\left(\mathbb{R}^{\mathbb{N}}\right)^{*}$. So, we can consider that each $A_{n}$ is independent of the second variable.

We apply Lemma 2.2 to each $A_{n}$ and obtain $A_{n}^{\prime} \in \mathscr{A} \mathscr{C}(X), A_{n}^{\prime}$ closed, $A_{n}^{\prime} \cap C \subseteq$ $A_{n} \cap C$ and $e^{*}\left(\sigma(u)\left(A_{n}^{\prime} \cap C\right)\right)+\varepsilon / 2^{n+1}>e^{*}\left(\sigma(u)\left(A_{n} \cap C\right)\right)$ for every $n$. Let $B_{n}=A_{1}^{\prime} \cap \cdots \cap A_{n}^{\prime}$. Then $e^{*}\left(\sigma(u)\left(B_{n} \cap C\right)\right)>\varepsilon / 2$ for every $n$. An application of Lemma 2.1 lets us find a sequence of positive numbers $\left\{M_{j}\right\}_{j}$ with the following property: for every $n, B_{n} \cap C \cap K \neq \emptyset$ where $K=\bigcap_{j=1}^{\infty}\left\{x \in X:\left|e_{j}^{*}(x)\right| \leq M_{j} e^{*}(x)\right\}$. This implies that $K_{n}=B_{n} \cap K \cap\left\{x \in X: e^{*}(x)=1\right\}$ is not empty. Observe that $K_{n}$ is of the form $K_{n}=L_{n} \times \mathbb{R}^{I^{\prime}}$ for a compact set $L_{n}$ in $\mathbb{R}^{\mathbb{N}}$. Since $\left\{K_{n}\right\}_{n}$ is decreasing, there is a point $x \in \bigcap_{n} K_{n} \subseteq \bigcap_{n} A_{n}$, a contradiction. This shows that $\sigma(u)$ is countably additive.

In the light of Theorem 2.3, if $u$ is a conical measure on a complete weak space $X$, one can consider the $\sigma$-algebra generated by $\mathscr{A} \mathscr{C}(X)$ which will be denoted by $\Sigma C(X)$. Since $\sigma(u)$ is weakly countably additive and has relatively weakly compact range, it has a unique extension to a countably additive vector measure on $\Sigma C(X)$, which we denote by $\tilde{\sigma}(u)$ and still satisfies $\Delta(\tilde{\sigma}(u))=u$.

If $X$ is not weakly complete, the measure $\sigma(u)$ is not necessarily countably additive even if $u=\Delta(F)$ for $F$ a countably additive vector measure (see Section 3). Nevertheless, if $u$ is a conical measure on any locally convex space $X$ such that $K_{u} \subseteq X$, as explained in Section 1, $u$ can also be viewed as a conical measure on $\widehat{X}$, the weak completion of $X$. In this setting we can consider the $\sigma$-algebra $\Sigma C(\widehat{X})$ on which $\tilde{\sigma}(u)$ is countably additive and has range inside $K_{u} \subseteq X$. So, if $(\Delta, \mathscr{M})=(\widehat{X}, \Sigma C(\widehat{X}))$ we get

COROLLARY 2.4. Let $X$ be a Hausdorff locally convex space. There exists a measurable space $(\triangle, \mathscr{M})$ with the following property: if $u$ is a conical measure on $X$ such that $K_{u} \subseteq X$, then there is a countably additive vector measure $F: \mathscr{M} \rightarrow X$ whose associated conical measure is $u$.

This corollary contains the result of Kluvanek [K1, Theorem 5] which states that every conical measure $u$ such that $K_{u} \subseteq X$ is the conical measure associated to a countably additive measure defined on a $\sigma$-algebra. The improvement here is that the measurable space is the same for every conical measure.

Using the same ideas we can finish the proof of Theorem 1.2. There is a natural identification between $\mathscr{A} \mathscr{C}(X)$ and $\mathscr{A} \mathscr{C}(\widehat{X})$ given by the Boolean isomorphism $M \mapsto \widehat{M}$ defined by the property that, for every $x^{*} \in X^{*}$, we have $\widehat{A_{x^{*}}}=\left\{\hat{x} \in \widehat{X}: x^{*}(\hat{x})>0\right\}$, 
if $A_{x^{*}}=\left\{x \in X: x^{*}(x)>0\right\}$. When considering a conical measure $u$ on $X$ such that $K_{u} \subset X$, if we call $\hat{u}$ the conical measure $u$ viewed on $\widehat{X}$, this identification respects the construction of the measures associated to $u$ in Theorem 1.2 in the sense that $\sigma(\hat{u})(\widehat{M})=\sigma(u)(M)$, for every $M \in \mathscr{A} \mathscr{C}(X)$.

Despite $\sigma(\hat{u})$ being countably additive, $\sigma(u)$ may not be so; the reason for this is that there can exist a decreasing sequence $\left(A_{n}\right)$ in $\mathscr{A} \mathscr{C}(X)$ such that $\bigcap_{n} A_{n}=\emptyset$, but $\bigcap_{n} \widehat{A}_{n} \neq \emptyset$. This is impossible if the sequence $\left(A_{n}\right)$ is contained in $\mathscr{A} \mathscr{C}(X, Z)$ for some finite dimensional subspace $Z$ of $X^{*}$; since if $\hat{x}: X^{*} \rightarrow \mathbb{R}$ is a linear function, there exists $x \in X$ such that $x^{*}(x)=\hat{x}\left(x^{*}\right)$ for every $x^{*} \in Z$, thus, if $\hat{x}$ belongs to $\bigcap_{n} \widehat{A_{n}}$, we have $x \in \bigcap_{n} A_{n}$. Therefore, since $\sigma(\hat{u})$ is countably additive in $\mathscr{A} \mathscr{C}(\widehat{X}, Z)$, we obtain

COROLLARY 2.5. Let $X$ be a Hausdorff locally convex space. If $u$ is a conical measure on $X$ such that $K_{u} \subseteq X$, and $\sigma(u)$ is the measure constructed in the proof of Theorem 1.2, then, for every finite dimensional subspace $Z$ of $X^{*}$, the restriction of $\sigma(u)$ to $\mathscr{A} \mathscr{C}(X, Z)$ is countably additive.

The last aim of this section is to give a new proof of a localization theorem for conical measures in complete weak spaces due to Kluvánek [K2]. We say that a conical measure on $X$ is localizable if there exist a subset $S$ of $X$, a $\sigma$-algebra $\Sigma$ on $S$, and a positive measure $\mu$ on $\Sigma$ such that, for every $z^{*} \in h(X)$, its restriction to $S$ is $\mu$-integrable, and $u\left(z^{*}\right)=\int_{S} z^{*} d \mu$. In this case we say that $u$ is localized on $(S, \Sigma)$, and $\mu$ is a localization of $u$.

We use the following proposition about localization of $u$ in a general locally convex space $X$ when $\sigma(u)$ is countably additive. In this case, $\sigma(u)$ can be extended as a countably additive measure $\tilde{\sigma}(u)$ to the $\sigma$-algebra $\Sigma C(X)$ generated by $\mathscr{A} \mathscr{C}(X)$. If $x_{0}^{*} \in X^{*}$ and $\Pi=\left\{x \in X: x_{0}^{*}(x)=1\right\}$, the $\sigma$-algebra in $\Pi$ generated by the sets $\{A \cap \Pi: A \in \mathscr{A} \mathscr{C}(X)\}$ is $\Sigma_{\Pi}=\{C \cap \Pi: C \in \Sigma C(X)\}$, and coincides with the $\sigma$-algebra generated in $\Pi$ by the elements of $X^{*}$ (or $h(X)$ ). Observe that, if $x^{*} \in X^{*}$, and $\alpha \in \mathbb{R}$; then $\left\{x \in \Pi: x^{*}(x)>\alpha\right\}=\Pi \cap\left\{x \in X:\left(x^{*}-\alpha x_{0}^{*}\right)(x)>0\right\}$. We have

PROPOSITION 2.6. Let $X$ be a Hausdorff locally convex space and $u \in M^{+}(X)$ such that $K_{u} \subseteq X$ and $\sigma(u)$ is countably additive. For $x_{0}^{*} \in X^{*}$, let $A$ be the cone $A=\left\{x \in X: x_{0}^{*}(x)>0\right\}$ and $\Pi=\left\{x \in X: x_{0}^{*}(x)=1\right\}$. There exists a positive finite countably additive measure $\mu$ on $\Sigma_{\Pi}$ such that $z^{*} \in L^{1}(\mu)$ and

$$
u_{A}\left(z^{*}\right)=\int_{\Pi} z^{*} d \mu, \quad \text { for every } z^{*} \in h(X) .
$$

In this case, we have, for every $x^{*} \in X^{*}$, and every $C \in \Sigma C(X)$,

$$
x^{*}(\tilde{\sigma}(u)(C \cap A))=\int_{C \cap \Pi} x^{*} d \mu .
$$


PROOF. Observe that the sets in $\Sigma C(X)$ are also cones, and therefore $C_{1} \cap \Pi=$ $C_{2} \cap \Pi$ if and only if $C_{1} \cap A=C_{2} \cap A$, for $C_{1}, C_{2} \in \Sigma C(X)$. This allows us to define $\mu(C \cap \Pi)=x_{0}^{*}(\tilde{\sigma}(u)(C \cap A))$, for every $C \in \Sigma C(X)$. It is easy to check that $\mu$ is a positive finite countably additive measure on $\Sigma_{\Pi}$.

If $z^{*} \in h(X)$, then the restriction of $z^{*}$ to $\Pi$ is $\Sigma_{\Pi}$-measurable. Let us observe that it is enough to prove (6) for $z^{*} \in h^{+}(X)$. Moreover, thanks to the monotone convergence theorem, we can assume that $z^{*}$ is bounded on $\Pi$, since, for every $z^{*} \in h^{+}(X)$,

$$
u_{A}\left(z^{*}\right)=\lim _{n} u\left(\left(n x_{0}^{*} \wedge z^{*}\right)^{+}\right)=\lim _{n} u_{A}\left(\left(n x_{0}^{*} \wedge z^{*}\right)^{+}\right),
$$

and $0 \leq\left(\left(n x_{0}^{*} \wedge z^{*}\right)^{+}\right) \leq n$ on $\Pi$. For this $z^{*}$, there exists $\left\{C_{j}\right\}_{j \in J}$ a finite partition of $X$ into elementary cones, such that $z^{*}$ coincides with some $y_{j}^{*} \in X^{*}$ in $C_{j}$. Since $u_{A}=\sum_{j}\left(u_{A}\right)_{C_{j}}=\sum_{j} u_{A \cap C_{j}}$, it is enough to prove

$$
\int_{\Pi \cap C} y^{*} d \mu=u_{C}\left(y^{*}\right)
$$

for every elementary cone $C \subseteq A$, and every $y^{*} \in X^{*}$ which is bounded on $C \cap \Pi$. To show this fact, remember that $u_{C}\left(y^{*}\right)=y^{*}\left(r\left(u_{C}\right)\right)=y^{*}(\sigma(u)(C))$. So, (8) holds for $y^{*}=x_{0}^{*}$, since, for $C \subseteq A$,

$$
\int_{\Pi \cap C} x_{0}^{*} d \mu=\int_{\Pi \cap C} d \mu=x_{0}^{*}(\sigma(u)(C))=u_{C}\left(x_{0}^{*}\right) .
$$

If $C \subseteq A$ and $y^{*}$ is bounded on $C \cap \Pi$, take $\varepsilon>0$ and consider, for every $j \in \mathbb{Z}$, the elementary cone

$$
C_{j}=C \cap\left\{x \in X:(j-1) \varepsilon x_{0}^{*}(x) \leq y^{*}(x)<j \varepsilon x_{0}^{*}(x)\right\} .
$$

The family $\left\{C_{j}\right\}_{j \in \mathbb{Z}}$ is a partition of $C$. The boundedness of $y^{*}$ on $C \cap \Pi$ implies that only finitely many $C_{j}$ 's are not empty, and we have $u_{C}\left(y^{*}\right)=\sum_{j} u_{C_{j}}\left(y^{*}\right)$. On $C_{j}$, we have $j \varepsilon x_{0}^{*}-y^{*}=\left|j \varepsilon x_{0}^{*}-y^{*}\right|$, and, by Lemma 1.5 and (9),

$$
u_{C_{j}}\left(y^{*}\right) \leq j \varepsilon u_{C_{j}}\left(x_{0}^{*}\right)=j \varepsilon \int_{\Pi \cap C_{j}} x_{0}^{*} d \mu \leq \int_{\Pi \cap C_{j}} y^{*} d \mu+\varepsilon \int_{\Pi \cap C_{j}} x_{0}^{*} d \mu .
$$

Analogously one can prove

$$
u_{C_{j}}\left(y^{*}\right) \geq \int_{\Pi \cap C_{j}} y^{*} d \mu-\varepsilon \int_{\Pi \cap C_{j}} x_{0}^{*} d \mu .
$$

Summing up for $j \in \mathbb{Z}$, we obtain

$$
\left|u_{C}\left(y^{*}\right)-\int_{\Pi \cap C} y^{*} d \mu\right| \leq \varepsilon \int_{\Pi \cap C} x_{0}^{*} d \mu=\varepsilon \mu(\Pi \cap C),
$$


and (8) is proved since $\varepsilon$ was arbitrary.

Let us prove the last assertion in the statement. Observe that both terms in equality (7) define real measures on $\Sigma C(X)$, so it is enough to prove (7) for $C$ in $\mathscr{A} \mathscr{C}(X)$, an algebra generating $\Sigma C(X)$. Moreover, it suffices to consider that $C$ is an elementary cone. Then $x^{*}(\tilde{\sigma}(u)(C \cap A))=x^{*}(\sigma(u)(C \cap A))=u_{C \cap A}\left(x^{*}\right)=\left(u_{A}\right)_{C}\left(x^{*}\right)$. Finally, applying the remark after Lemma 1.4 to identity (6), we know that $\left(u_{A}\right)_{C}\left(x^{*}\right)=$ $\int_{C \cap n} x^{*} d \mu$, and the proof of (7) is finished.

If $E$ is a linear subspace of the locally convex space $X$, it is clear that, for every $z^{*} \in h(X)$, the restriction $\left.z^{*}\right|_{E}$ belongs to $h(E)$. Conversely, as a consequence of the Hahn-Banach theorem, every $f \in h(E)$ can be extended to $X$ as an element of $h(X)$. In this way $M^{+}(E)$ can be viewed as a subset of $M^{+}(X)$. In the following lemma, whose easy proof is left to the reader, this situation is explained.

LEMMA 2.7. Let $X$ be a locally convex space, and $E$ a linear subspace of $X$. Given a conical measure $v \in M^{+}(E)$ and defining $u\left(z^{*}\right)=v\left(\left.z^{*}\right|_{E}\right)$ for every $z^{*} \in h(X)$, we obtain a conical measure $u \in M^{+}(X)$.

Conversely, if $u \in M^{+}(X)$ has the property that $u\left(z^{*}\right)=0$, for every $z^{*} \in h(X)$ such that $\left.z^{*}\right|_{E}=0$, then there exists an unique conical measure $\widehat{u} \in M^{+}(E)$ such that $u\left(z^{*}\right)=\widehat{u}\left(\left.z^{*}\right|_{E}\right)$, for every $z^{*} \in h(X)$. Moreover, for every elementary cone $A$ in $X$ we have $\widehat{u}_{A}=\widehat{u}_{A \cap E}$.

We can now give our proof of the next theorem due to Kluvánek [K2]. We are going to use the same measure space as Kluvánek uses to localize conical measures on $\mathbb{R}^{I}$ : the disjoint union $\Omega$ of certain measure spaces $\left\{\Omega_{\alpha}\right\}_{\alpha}$. We decompose first the conical measure as a sum of conical measures $u_{\alpha}$ such that each $u_{\alpha}$ can be localized on each $\Omega_{\alpha}$. Here is the difference with Kluvánek's method: he first extends the conical measure as a Daniell integral, then he decomposes this Daniell integral and localizes each component on the spaces $\Omega_{\alpha}$. The decomposition of the conical measure is easier since it is a functional acting on a smaller space of functions. The decomposition of the Daniell integral is not so clear and the inductive arguments do not appear to be completely correct in Kluvánek's paper.

Another proof of this result was given by Becker [B, Theorem 21]. We are indebted to the referee for pointing out this reference to us.

THEOREM 2.8. Let $X$ be a complete weak space. There exist a set $\Omega \subset X$, and a $\sigma$-algebra $\Sigma$ on $\Omega$ such that every conical measure on $X$ can be localized in $(\Omega, \Sigma)$.

PROOF. If $X$ is a complete weak space then there exists an index set $l$ such that, up to topological vector isomorphism, $X=\mathbb{R}^{l}$. Without loss of generality, we can assume that $I$ is a closed interval of ordinal numbers with 0 as the least element, and $\gamma$ as the greatest one; that is, $I=\{\alpha$ ordinal: $0 \leq \alpha \leq \gamma\}$. 
For every $\alpha \in I$, let $e_{\alpha}^{*}$ be the $\alpha$-th coordinate functional on $X$; that is, $e_{\alpha}^{*}\left(\left(x_{i}\right)_{i \in I}\right)=$ $x_{\alpha}$, for every $\left(x_{i}\right)_{i \in l} \in X$. We consider, for every $\alpha \in I$, the following subsets of $X$ :

$$
\begin{gathered}
C_{\alpha}=\left\{x \in X: e_{\alpha}^{*}(x)=0\right\}, \quad A_{\alpha}^{+}=\left\{e_{\alpha}^{*}>0\right\}, \quad A_{\alpha}^{-}=\left\{e_{\alpha}^{*}<0\right\}, \\
\Pi_{\alpha}^{+}=\left\{e_{\alpha}^{*}=1\right\}, \quad \Pi_{\alpha}^{-}=\left\{e_{\alpha}^{*}=-1\right\}, \\
E_{\alpha}=\bigcap_{\beta \in I, \beta<\alpha} C_{\beta}=\left\{x=\left(x_{i}\right)_{i \in I} \in X: x_{i}=0, \text { for every } i<\alpha\right\},
\end{gathered}
$$

(where we understand $E_{0}=X$, and we also use $E_{\gamma+1}=\{0\}$ ),

$$
\Omega_{\alpha}^{+}=\Pi_{\alpha}^{+} \cap E_{\alpha}, \quad \Omega_{\alpha}^{-}=\Pi_{\alpha}^{-} \cap E_{\alpha}, \quad \text { and } \quad \Omega_{\alpha}=\Omega_{\alpha}^{+} \cup \Omega_{\alpha}^{-} .
$$

On each $\Omega_{\alpha}$ we consider the $\sigma$-algebra $\Sigma_{\alpha}$ generated by $X^{*}$ (or by $h(X)$, which is the same); the least $\sigma$-algebra on $\Omega_{\alpha}$ for which, the restriction to $\Omega_{\alpha}$ of every $x^{*} \in X^{*}$ is measurable. Observe that the sets $\Omega_{\alpha}$ 's are pairwise disjoint. Finally consider $\Omega=\bigcup_{\alpha \in I} \Omega_{\alpha}$, and the $\sigma$-algebra $\Sigma=\left\{B \subset \Omega: B \cap \Omega_{\alpha} \in \Sigma_{\alpha}\right.$, for every $\left.\alpha \in I\right\}$ defined on $\Omega$.

We are going to prove that, given a conical measure $u$ on $X$, there exists a family $\left\{u_{\alpha}: \alpha \in I\right\}$ of conical measures such that,

$$
u\left(z^{*}\right)=\sum_{\alpha \in I} u_{\alpha}\left(z^{*}\right), \quad \text { for every } z^{*} \in h(X) ;
$$

and, for every $\alpha \in I$, there exists a finite positive measure $\mu_{\alpha}$ on $\left(\Omega_{\alpha}, \Sigma_{\alpha}\right)$ such that

$$
u_{\alpha}\left(z^{*}\right)=\int_{\Omega_{\alpha}} z^{*} d \mu_{\alpha}, \quad \text { for every } z^{*} \in h(X) .
$$

Once this is done, we can define $\mu(B)=\sum_{\alpha \in I} \mu_{\alpha}\left(B \cap \Omega_{\alpha}\right)$ for $B \in \Sigma$, and it is clear, since the series in (10) converges absolutely, that

$$
u\left(z^{*}\right)=\int_{\Omega} z^{*} d \mu, \quad \text { for every } z^{*} \in h(X) .
$$

Let us define first the conical measure $w_{\alpha}$, for $\alpha>0$, as the infimum in $M^{+}(X)$

$$
w_{\alpha}=\inf \left\{u_{C_{\beta_{1}} \cap \cdots \cap C_{\beta_{n}}}: n \in \mathbb{N}, \beta_{1}, \ldots, \beta_{n}<\alpha\right\},
$$

and $w_{0}=u$. Observe that, as the set where we are taking infimum is directed, we have for every $z^{*} \in h^{+}(X)$,

$$
w_{\alpha}\left(z^{*}\right)=\inf \left\{u_{C_{\beta_{1}} \cap \cdots \cap C_{\beta_{n}}}\left(z^{*}\right): n \in \mathbb{N}, \beta_{1}, \ldots, \beta_{n}<\alpha\right\} .
$$


Finally, we define the conical measure

$$
u_{\alpha}=\left(w_{\alpha}\right)_{A_{\alpha}^{+}}+\left(w_{\alpha}\right)_{A_{\alpha}^{-}} .
$$

In order to prove (11), let us see that $w_{\alpha}\left(z^{*}\right)=0$, if $z^{*}(x)=0$ for every $x \in E_{\alpha}$. Pick $z^{*} \in h^{+}(X) ; z^{*}$ depends on a finite number of coordinates $\beta_{1}, \ldots, \beta_{m}$, that we suppose ordered so that $\beta_{1}<\cdots<\beta_{n}<\alpha \leq \beta_{n+1}<\cdots<\beta_{m}$. Take $x \in C_{\beta_{1}} \cap \cdots \cap C_{\beta_{n}}$, and define $y=\left(y_{\beta}\right)_{\beta} \in X$ by $y_{\beta}=x_{\beta}$, if $\beta \geq \alpha$, and $y_{\beta}=0$, if $\beta<\alpha$. We have $y \in E_{\alpha}$, so $z^{*}(y)=0$, but we also have $x_{\beta_{j}}=y_{\beta_{j}}$ for every $j=1, \ldots, m$, therefore $z^{*}(x)=z^{*}(y)=0$. We have proved that $z^{*}=0$ on the elementary cone $C_{\beta_{1}} \cap \cdots \cap C_{\beta_{n}}$ which implies $u_{C_{\beta_{1}} \cap \cdots \cap C_{\beta_{n}}}\left(z^{*}\right)=0$ and therefore $w_{\alpha}\left(z^{*}\right)=0$.

We are then in a position to apply Lemma 2.7: there exists a conical measure $\widehat{w}_{\alpha} \in M^{+}\left(E_{\alpha}\right)$, such that $w_{\alpha}\left(z^{*}\right)=\widehat{w}_{\alpha}\left(\left.z^{*}\right|_{E_{\alpha}}\right)$. Observe that $E_{\alpha}$ is a closed subspace, so it is also a weak complete space and, by Theorem $2.3, \sigma\left(\widehat{w}_{\alpha}\right)$ is countably additive. We can use Proposition 2.6 for $x_{0}^{*}=e_{\alpha}^{*}$ and $x_{0}^{*}=-e_{\alpha}^{*}$ to obtain a measure $\mu_{\alpha}$ on $\Sigma_{\alpha}$ such that

$$
\left(\widehat{w}_{\alpha}\right)_{A_{\alpha}^{+} \cap E_{\alpha}}(f)=\int_{\Omega_{\alpha}^{+}} f d \mu_{\alpha}, \quad \text { for every } f \in h\left(E_{\alpha}\right) ;
$$

and the same with $\Omega_{\alpha}^{-}$. By Lemma 2.7 we also know that

$$
\left(w_{\alpha}\right)_{A_{\alpha}^{+}}\left(z^{*}\right)=\left(\widehat{w}_{\alpha}\right)_{A_{\alpha}^{+} \cap E_{\alpha}}\left(\left.z^{*}\right|_{E_{\alpha}}\right)=\int_{\Omega_{\alpha}^{+}} z^{*} d \mu_{\alpha}, \quad \text { for every } z^{*} \in h(X),
$$

which yields (11).

An application of Proposition 1.7 (d) gives $\left(w_{\alpha}\right)_{C_{\alpha}}=w_{\alpha+1}$ for every $\alpha \in I$. Thus we deduce

$$
w_{\alpha}=u_{\alpha}+w_{\alpha+1} .
$$

Let us prove, by transfinite induction, that for every $\alpha \leq \gamma+1$,

$$
u\left(z^{*}\right)=\sum_{\beta<\alpha} u_{\beta}\left(z^{*}\right)+w_{\alpha}\left(z^{*}\right), \quad \text { for every } z^{*} \in h^{+}(X) .
$$

If $\alpha$ is an ordinal limit, (13) is a consequence of $w_{\alpha}=\inf \left\{w_{\beta}: \beta<\alpha\right\}$ and the inductive hypothesis. If $\alpha=\alpha^{\prime}+1,(13)$ is a consequence of (12) and the inductive hypothesis.

To obtain (10) it remains to prove that $w_{\gamma+1}=0$, since (13) applies for $\alpha=\gamma+1$. If $z^{*} \in h^{+}(X)$ it depends on a finite number of coordinates, this implies that there exist $\beta_{1}, \ldots, \beta_{m} \in I$ such that $z^{*}(x)=0$, if $e_{\beta_{j}}^{*}(x)=0$, for $j=1, \ldots, n$. Then $z^{*}=0$ on $C_{\beta_{1}} \cap \cdots \cap C_{\beta_{n}}$ and, as $\beta_{1}, \ldots, \beta_{m}$ are admissible in the infimum defining $w_{\gamma+1}$, we have $w_{\gamma+1}\left(z^{*}\right) \leq u_{C_{\beta_{1}} \cap \cdots \cap C_{\beta_{n}}}\left(z^{*}\right)=0$. 


\section{Conical measures on Banach spaces}

In the rest of this paper we restrict ourselves to the case when $X$ is a Banach space. By $B_{X}$ we denote the unit ball of $X$. If $X$ is infinite dimensional, $X$ does not coincide with $\widehat{X}$ and, in general, for a conical measure $u$ on $X, \sigma(u)$ is not countably additive. In fact, we prove that this latter condition is equivalent to being the conical measure associated to a Pettis differentiable measure (see Theorem 3.3).

In the following example we show a situation where $\sigma(u)$ is not countably additive. Assume that the conical measure $u$ is the conical measure associated to a countably additive vector measure $F:(\Omega, \Sigma) \rightarrow X$. Let $\lambda$ be a control measure for $F$ (that is, a positive finite measure such that $\lambda(A)=0$ implies $F(A)=0$ [DU, page 14]) and, for $x^{*} \in X^{*}$, let $f_{x^{*}}=d\left(x^{*} F\right) / d \lambda$ be the Radon-Nikodym derivative of the real measure $x^{*} \circ F$ with respect to $\lambda$. If $H=\bigcap_{i \in I}\left\{x_{i}^{*}>0\right\} \cap\left(\bigcap_{i \in K}\left\{x_{i}^{*} \geq 0\right\}\right)$ is an elementary cone, let $\widehat{H} \in \Sigma$ be the set

$$
\widehat{H}=\left(\bigcap_{i \in I}\left\{f_{x_{i}^{*}}>0\right\}\right) \cap\left(\bigcap_{i \in K}\left\{f_{x_{i}^{*}} \geq 0\right\}\right)
$$

Then it is easy to show that $u_{H}=\Delta\left(F_{\widehat{H}}\right)$ and $\sigma(u)(H)=F(\widehat{H})$.

EXAMPLE 3.1. A $c_{0}$-valued countably additive vector measure $F$ with bounded variation such that $\sigma(\Delta(F))$ defined on $\mathscr{A} \mathscr{C}\left(c_{0}\right)$ is not countably additive.

ProOF. Let $m$ be the Lebesgue measure on $[0,1]$ and $\left\{r_{n}\right\}_{n}$ the sequence of Rademacher functions. Let $\left\{s_{n}\right\}_{n}$ be the sequence of functions $s_{1}=1$ and $s_{n+1}=r_{n}$, for $n \geq 1$. We define the vector measure $F$ on the $\sigma$-algebra of Lebesgue measurable sets of $[0,1]$ by

$$
F(A)=\left(\int_{A} s_{n}(t) d m(t)\right)_{n}
$$

We consider, for every integer $n \geq 2$, the set $C_{n} \in \mathscr{A} \mathscr{C}\left(c_{0}\right)$ defined by

$$
C_{n}=\left\{\left(x_{n}\right)_{n} \in c_{0}: x_{1}>0,\left|x_{2}\right| \geq x_{1}, \ldots,\left|x_{n}\right| \geq x_{1}\right\}
$$

It is easy to show that $\bigcap_{n \geq 2} C_{n}=\emptyset$ but $\widehat{C}_{n}=[0,1]$, for every $n$, since $s_{1}=1$ and $\left|s_{k}\right|=1$ for every $k, m$-almost everywhere. Then $\sigma(\Delta(F))\left(C_{n}\right)$ does not converges to 0 and $\sigma(\Delta(F))$ is not countably additive.

LEMMA 3.2. Let $X$ be a Banach space and $u \in M^{+}(X)$ satisfying $K_{u} \subseteq X$. Then there exists $x_{0}^{*} \in X^{*}$ such that $u_{C}=0$ where $C=\left\{x \in X: x_{0}^{*}(x)=0\right\}$. 
PROOF. Let $F:(\Omega, \Sigma) \rightarrow X$ be a countably additive vector measure on a $\sigma$-algebra $\Sigma$ such that $u=\Delta(F)$ and $\lambda$ a control measure for $F$. For this vector measure, there exists a Rybakov functional $x_{0}^{*} \in X^{*}$ (see [DU, page 268]); that is, $F$ is absolutely continuous with respect to $\left|x_{0}^{*} F\right|$. If $A=\left\{w \in \Omega:\left(d\left(x_{0}^{*} F\right) / d \lambda\right)(w)=0\right\}$, then $F_{A}=0$. But it is easy to show that $u_{C}=\Delta\left(F_{A}\right)$, so $u_{C}=0$.

THEOREM 3.3. Let $X$ be a Banach space and $u \in M^{+}(X)$ satisfying $K_{u} \subseteq X$. The following properties are equivalent:

(a) $\sigma(u)$ is countably additive.

(b) $x^{*} \circ \sigma(u)$ is countably additive for every $x^{*} \in X^{*}$.

(c) There exists a measure space $(\Omega, \Sigma, \mu)$ and $g: \Omega \rightarrow X$ Pettis integrable such that if $G$ is the indefinite integral with respect to $g$, then $\Delta(G)=u$.

In particular, any of the above conditions implies that $\tilde{\sigma}(u)$ has $\sigma$-finite variation.

PROOF. As explained in the introduction, the equivalence of (a) and (b) is due to the Orlicz-Pettis theorem and the fact that $K_{u}$ is a weakly compact set.

To show that (a) implies (c), we first recall that $\sigma(u)$ has a countably additive extension $\tilde{\sigma}(u)$ to $\Sigma C(X)$. Take $x_{0}^{*}$ as in previous lemma. If $A^{+}=\left\{x \in X: x_{0}^{*}(x)>0\right\}$, $A^{-}=\left\{x \in X: x_{0}^{*}(x)<0\right\}$ and $C=\left\{x \in X: x_{0}^{*}(x)=0\right\}$, then $u=u_{A^{+}}+u_{A^{-}}$. Let $\Pi^{+}=\left\{x \in X: x_{0}^{*}(x)=1\right\}, \Pi^{-}=\left\{x \in X: x_{0}^{*}(x)=-1\right\}, A=A^{+} \cup A^{-}$and $\Pi=\Pi^{+} \cup \Pi^{-}$. If $\mu(C \cap \Pi)=\left|x_{0}^{*} \circ \tilde{\sigma}(u)\right|(C \cap A)$ for every $C \in \Sigma C(X)$, using Proposition 2.6, we get that for every $z^{*} \in h(X)$,

$$
u\left(z^{*}\right)=u_{A^{+}}\left(z^{*}\right)+u_{A^{-}}\left(z^{*}\right)=\int_{\Pi} z^{*} d \mu
$$

and that for every $x^{*} \in X^{*}$ and every $C \in \Sigma C(X)$,

$$
x^{*}(\tilde{\sigma}(u)(C))=\int_{C \cap \Pi} x^{*} d \mu .
$$

If $\Omega=\Pi, \Sigma$ is the $\sigma$-algebra $\Sigma=\{C \cap \Pi: C \in \Sigma C(X)\}$ and $G$ is the vector measure on $\Sigma$ defined by $G(C \cap \Pi)=\tilde{\sigma}(u)(C \cap A)=\tilde{\sigma}(u)(C)$, then $g(x)=x$ is a Pettis derivative of $G$ with respect to $\mu$ and $\Delta(G)=u$.

Let us suppose now that (c) holds. In this case, it is easy to show that $\Delta(G)\left(z^{*}\right)=$ $\int z^{*} \circ g d \mu$ for every $z^{*} \in h(X)$ and that $(\Delta(G))_{C}=\Delta\left(\left.G\right|_{\left(g^{-1}(C)\right\}}\right)$ for every $C \in$ $\mathscr{A} \mathscr{C}(X)$. This implies that $\sigma(u)(C)=G\left(g^{-1}(C)\right)$. So if $C_{n}$ is a sequence in $\mathscr{A} \mathscr{C}(X)$ decreasing to the empty set, $g^{-1}\left(C_{n}\right)$ also decreases to the empty set and $\sigma(u)\left(C_{n}\right)$ converges to 0 . This shows (a).

To prove the last assertion of this theorem, remember that every Pettis differentiable vector measure has $\sigma$-finite variation (see [M]). Then the vector measure defined in (c) has $\sigma$-finite variation. This shows that any other countably additive vector measure 
$F$ satisfying $\Delta(F)=u$, has $\sigma$-finite variation (see [RR]). In particular, $\tilde{\sigma}(u)$ has $\sigma$-finite variation.

If $(X,\|\cdot\|)$ is a Banach space with bidual $X^{* *}, X$ is a dense subset of $X^{* *}$ when considering the weak* topology $w^{*}=\sigma\left(X^{* *}, X^{*}\right)$, so we can identify $h(X,\|\cdot\|)$ with $h\left(X^{* *}, w^{*}\right)$. Let $u$ be a conical measure on $X$ such that $K_{u} \subseteq X$. The same construction of Theorem 1.2 is valid taking $\left(X^{* *}, w^{*}\right)$ instead of $(X,\|\cdot\|)$. If we take $\tau(u)(A)=r\left(u_{A}\right)$ for every $A \in \mathscr{A} \mathscr{C}\left(X^{* *}, w^{*}\right)$, then $\tau(u)$ defines a finitely additive vector measure with values in $X$. We prove in Theorem 3.4 that $\tau(u)$ is countably additive if and only if $u$ is the conical measure associated to a countably additive vector measure $F$ on a $\sigma$-algebra with $\sigma$-finite variation.

Whenever $\tau(u)$ is countably additive, we denote by $\tilde{\tau}(u)$ the countably additive extension of $\tau(u)$ defined on the $\sigma$-algebra $\Sigma C\left(X^{* *}, w^{*}\right)$. There are examples of countably additive vector measures $F: \mathscr{F} \rightarrow X$ on a field $\mathscr{F}$ which do not have $\sigma$ finite variation, but having an extension to the $\sigma$-algebra generated by $\mathscr{F}$ with $\sigma$-finite variation (see $[R]$ ). This is the reason why we consider the vector measure $\tilde{\tau}(u)$ in the following lemma instead of $\tau(u)$.

Following the same line as Theorem 3.3 with $\tau(u)$ instead of $\sigma(u)$, we get the following theorem.

THEOREM 3.4. Let $X$ be a Banach space and $u \in M^{+}(X)$ satisfying $K_{u} \subseteq X$. The following properties are equivalent:

(a) $\tau(u)$ is countably additive.

(b) $\tau(u)$ is countably additive and $\tilde{\tau}(u)$ has $\sigma$-finite variation.

(c) Every countably additive vector measure $F$ on a $\sigma$-algebra such that $u=\Delta(F)$ has $\sigma$-finite variation.

$\left(c^{\prime}\right)$ There exists a countably additive vector measure $F$ on a $\sigma$-algebra such that $u=\Delta(F)$ has $\sigma$-finite variation.

(d) There exists a finite measure space $(\Omega, \Sigma, \mu)$, a countably additive vector measure $F$ on $(\Omega, \Sigma)$ with $\Delta(F)=u$ and $f: \Omega \rightarrow X^{* *}$ weak $k^{*}$ integrable such that

$$
\left\langle x^{*}, F(A)\right\rangle=\int_{A} x^{*} \circ f d \mu \text { for every } x^{*} \in X^{*} .
$$

PROOF. Let us suppose that (a) holds and that $F:(\Omega, \Sigma) \rightarrow X$ is a countably additive vector measure with non $\sigma$-finite variation such that $u=\Delta(F)$. Let $\lambda=$ $\left|x_{0}^{*} \circ F\right|$ be a control measure for it (where $x_{0}^{*}$ is a Rybakov functional) and $g_{x^{*}}=$ $d\left(x^{*} F\right) / d \lambda$ for $x^{*} \in X^{*}$. We recall that a vector measure in those conditions has $\sigma$-finite variation if and only if the lattice supremum $\sup _{x^{*} \in B_{x^{*}}}\left|g_{x^{*}}\right|$ exists in $L^{0}(\lambda)$. Since $F$ does not have $\sigma$-finite variation, there exists a set $A \in \Sigma$ with $\lambda(A)>0$ such that, taking supremum in the lattice $L^{0}(\lambda), \sup _{x^{*} \in B_{x^{*}}}\left|g_{x^{*}}\right|=+\infty$ on $A$. 
Then there is a sequence $\left\{x_{n}^{*}\right\}_{n \geq 1} \subseteq B_{X^{*}}$ such that $\sup _{n \geq 1}\left|g_{x_{n}^{*}}(x)\right|=+\infty \lambda$-almost everywhere on $A$. We set $g_{n}=g_{x_{n}^{*}}$, for $n \geq 0$. There exists an increasing sequence of positive integers $\left(n_{k}\right)_{k}$ such that if $B_{k}=\left\{\omega \in \Omega: \sup _{1 \leq i \leq n_{k}}\left|g_{i}(\omega)\right| \geq k\right\}$ and $B=\bigcap_{k=1}^{\infty} B_{k}$ then $0<\lambda(B)=\left|x_{0}^{*} \circ F\right|(B)$. We can assume that $\left(x_{0}^{*} \circ F\right)^{+}(B)>0$ (if not, consider $-x_{0}^{*}$ instead of $x_{0}^{*}$ ). Taking

$$
D_{k}=\left\{x \in X^{* *}: x_{0}^{*}(x)>0, \sup \left\{\left|x_{1}^{*}(x)\right|,\left|x_{2}^{*}(x)\right|, \ldots,\left|x_{n_{k}}^{*}(x)\right|\right\} \geq k x_{0}^{*}(x)\right\},
$$

each $D_{k}$ belongs to the algebra generated by the elementary cones and $C_{k}=\bigcap_{l=1}^{k} D_{l}$ is a decreasing sequence in that algebra with $\bigcap_{k} C_{k}=\emptyset$ and $\bigcap_{k} \widehat{C}_{k}=\left(\bigcap_{k} B_{k}\right) \cap\{w \in$ $\Omega:\left(d x_{0}^{*} F / d \lambda\right)(w)>0$ \} which implies

$$
\lim _{k \rightarrow \infty} x_{0}^{*}\left(\tau(\Delta(F))\left(C_{k}\right)\right)=\left(x_{0}^{*} \circ F\right)^{+}(B)>0 .
$$

We deduce that $\tau(\Delta(F))=\tau(u)$ is not countably additive, a contradiction. This shows that (a) implies (c).

Since (a) implies (c), we have that (a) implies (b), and it is clear that (b) implies (a).

It is obvious that (c) implies ( $\left.c^{\prime}\right)$ using Corollary 2.4 .

We show now that $\left(\mathrm{c}^{\prime}\right)$ implies (d). Suppose that there exists a countably additive vector measure $F:(\Omega, \Sigma) \rightarrow X$ such that $u=\Delta(F)$ and $F$ has $\sigma$-finite variation. Let $\mu$ be a finite control measure for $F$. Without loss of generality we can assume that $(\Omega, \Sigma, \mu)$ is a complete measure space. A result due to Rybakov in [Ry] (see also $\left[\mathrm{M}\right.$, Theorem 0]) lets us find a weak* integrable function $f: \Omega \rightarrow X^{* *}$ such that

$$
\left\langle x^{*}, F(A)\right\rangle=\int_{A} x^{*} \circ f d \mu \quad \text { for every } x^{*} \in X^{*} .
$$

This proves (d).

To show that (d) implies (a), we follow the same ideas as in Theorem 3.3.

Since on every infinite dimensional Banach space there is a vector measure with non $\sigma$-finite variation (see $[\mathrm{JK}]$ ), we get that in every infinite dimensional Banach space there is a conical measure $u$ such that $\tau(u)$ is not countably additive.

\section{References}

[B] R. Becker, 'Sur l'intégrale de Daniell', Rev. Roumaine Math. Pures Appl. 26 (1981), 189-206.

[BB] K. P. S. Bhaskara Rao and M. Bhaskara Rao, Theory of charges (Academic Press, London, 1983).

[C] C. H. Choquet, Lectures on Analysis vol. I, II, III (Benjamin, New York, 1969).

[DU] J. Diestel and J. J. Uhl Jr., Vector Measures, Amer. Math. Soc. Surveys 15 (Amer. Math. Soc., Providence, R. I., 1977). 
[JK] L. Janicka and N. J. Kalton, 'Vector measures of infinite variation', Bull. Acad. Polon. Sci. Ser. Sci. Math. Astron. et Phys. 25 (1977), 239-241.

[K1] I. Kluvánek, 'Characterization of the closed convex hull of the range of a vector measure', J. Funct. Anal. 21 (1976), 316-329.

[K2] —_. 'Conical measures and vector measures', Ann. Inst. Fourier (Grenoble) 217 (1977), 83-105.

[KK] I. Kluvánek and G. Knowles, Vector measures and control systems (North-Holland, Amsterdam, 1975).

[M] K. Musial, 'The weak Radon-Nikodym property in Banach spaces', Studia Math. 64 (1979), 151-173.

[R] L. Rodríguez Piazza, Rango y propiedades de medidas vectoriales. Conjuntos p-Sidon p.s. (Ph. D. Thesis, Universidad de Sevilla, 1991).

[RR] L. Rodríguez Piazza and M. C. Romero-Moreno, 'Conical measures and properties of a vector measure determined by its range', Studia Math. 125 (1997), 255-270.

[Ry] V. I. Rybakov, 'On vector measures', Izv. Vyssh. Uchebn. Zaved. Mat. 79 (1968), 92-101.

Departamento de Análisis Matemático

Facultad de Matemáticas

Universidad de Sevilla

Aptdo. 1160

Sevilla 41080

Spain

e-mail: piazza@cica.es, mcromero@cica.es 